\title{
Review Article \\ Role of Electroencephalography in Presurgical Evaluation of Temporal Lobe Epilepsy
}

\author{
Seetharam Raghavendra, ${ }^{1}$ Javeria Nooraine, ${ }^{1}$ and Seyed M. Mirsattari ${ }^{2}$ \\ ${ }^{1}$ Department of Neurology, Vikram Hospital, Bangalore 560052, India \\ ${ }^{2}$ Departments of Clinical Neurological Sciences, Medical Imaging, Medical Biophysics, and Psychology, \\ University of Western Ontario, London, ON, Canada N6A 5A5
}

Correspondence should be addressed to Seyed M. Mirsattari, smirsat2@uwo.ca

Received 5 September 2011; Revised 18 January 2012; Accepted 28 June 2012

Academic Editor: Warren T. Blume

Copyright (C) 2012 Seetharam Raghavendra et al. This is an open access article distributed under the Creative Commons Attribution License, which permits unrestricted use, distribution, and reproduction in any medium, provided the original work is properly cited.

Surgery remains a therapeutic option for patients with medically refractory epilepsy. Comprehensive presurgical evaluation includes electroencephalography (EEG) and video EEG in identifying patients who are likely to benefit from surgery. Here, we discuss in detail the utility of EEG in presurgical evaluation of patients with temporal lobe epilepsy along with illustrative cases.

\section{Introduction}

Temporal lobe epilepsy (TLE) is the most common form of epilepsy worldwide. Anterior temporal lobectomy (ATL) for medically refractory TLE secondary to mesial temporal sclerosis (MTS) is the most commonly performed surgical procedure in many of the comprehensive epilepsy management centres. Surgery is ideally directed towards complete seizure freedom without or with very minimal cognitive or functional deficits. Wiebe et al. in 2001 published the only randomised control study demonstrating the effectiveness of surgery in adults with medically refractory TLE [1]. Here, we would like to emphasise that the art of presurgical workup is to effectively use all the clinical, imaging, and electrophysiological information to localize the seizure onset zone (SOZ) and the epileptic network. In this paper, the electroencephalography (EEG) aspects of TLE with relevance to surgery are discussed with illustrative cases (see Table 1).

\section{Surface EEG}

Electrophysiological assessment remains the cornerstone for patients with TLE [10]. Standard EEG with 10-20 system provides limited coverage of the temporal regions detecting only about $58 \%$ of temporal spikes or interictal epileptiform discharges (IEDs). Additional electrodes help in increasing this yield $[11,12]$. Silverman's electrodes (T1 and T2, placed at posterior one-third and anterior two-thirds of a line connecting the outer canthus of the eye and the tragus) are often used to record from the anterior-basal areas of the temporal lobes [13-16]. Mandibular notch, nasopharyngeal (NP), sphenoidal (SP), and foramen ovale (FO) electrodes also help similarly.

NP recordings are cumbersome and provide little information over the well-tolerated routine anterior temporal and ear recordings especially with regard to anterior temporal IEDs $[17,18]$. However, NP recordings have increased sensitivity for IEDs arising from mesiobasal temporal regions (increasing IEDs identification by 25\%) [19]. FO electrodes offer a unique opportunity for simultaneous intracranial and surface EEG recording without breach of the skull. They may lateralize seizures in adults and children with mesial TLE when scalp ictal EEG fails [20-22]. It serves as intermediary between surface and invasive recordings.

The utility of SP electrodes remains debated. In TLE, fluoroscopic placement of SP electrodes below foramen ovale increases the sensitivity and interrater agreement for recognizing IEDs and ictal rhythms $[18,23]$. In asymmetric onset ictal scalp EEG recordings, ictal changes may be 


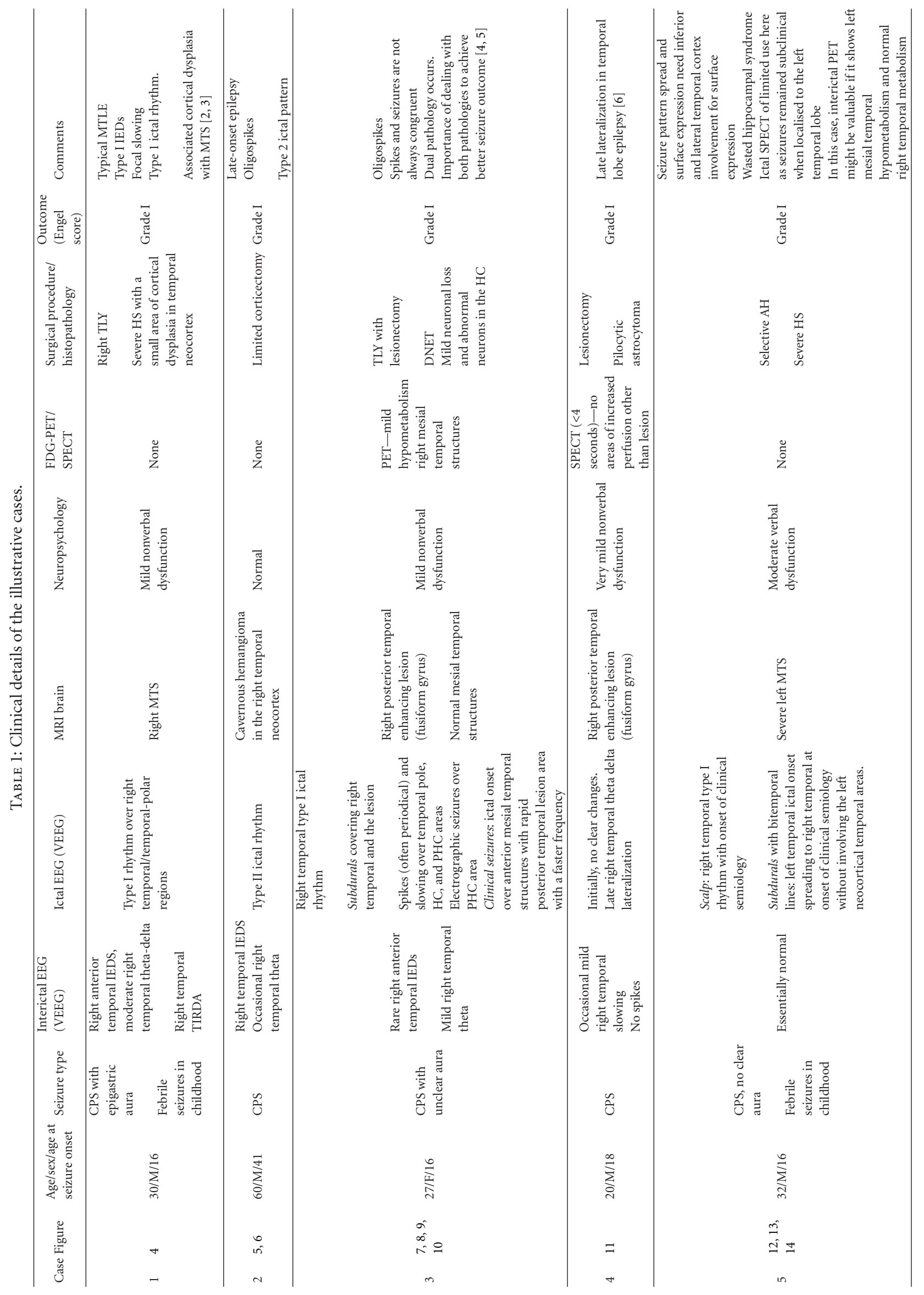




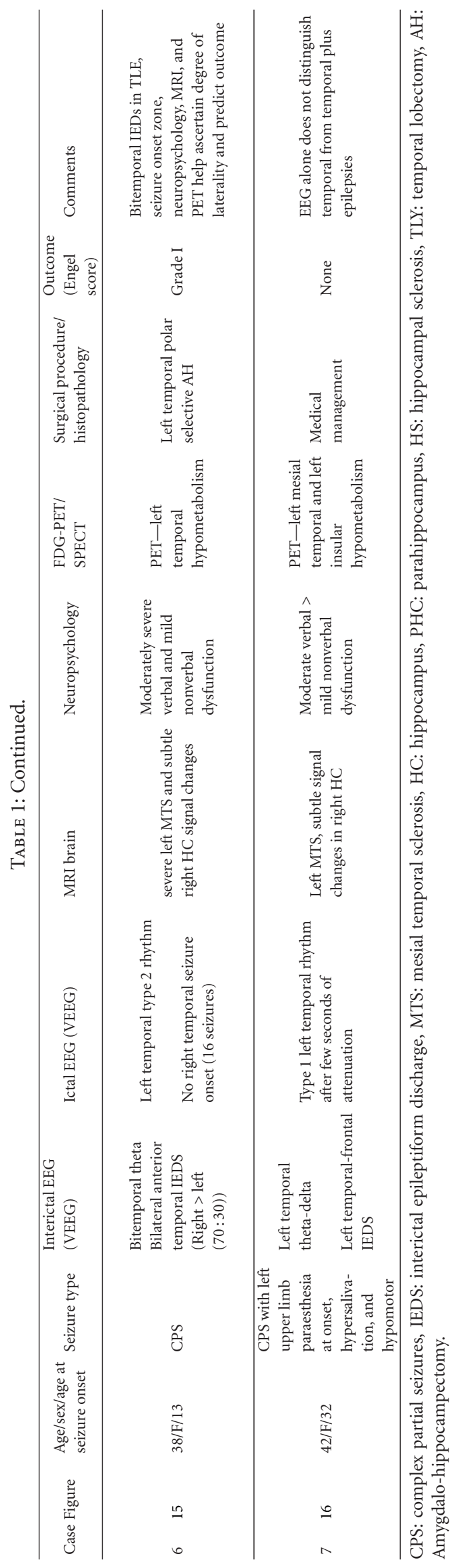




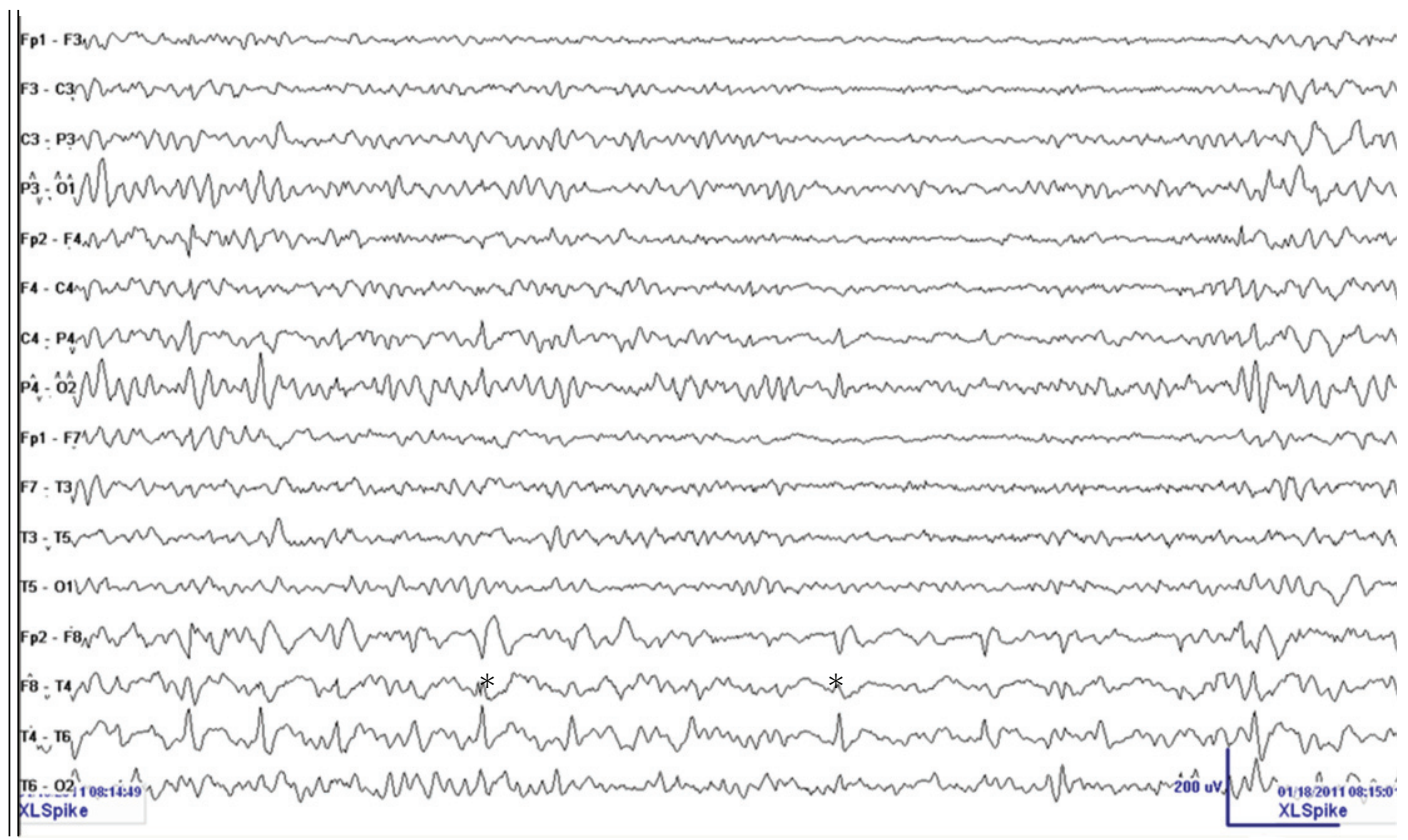

(a)

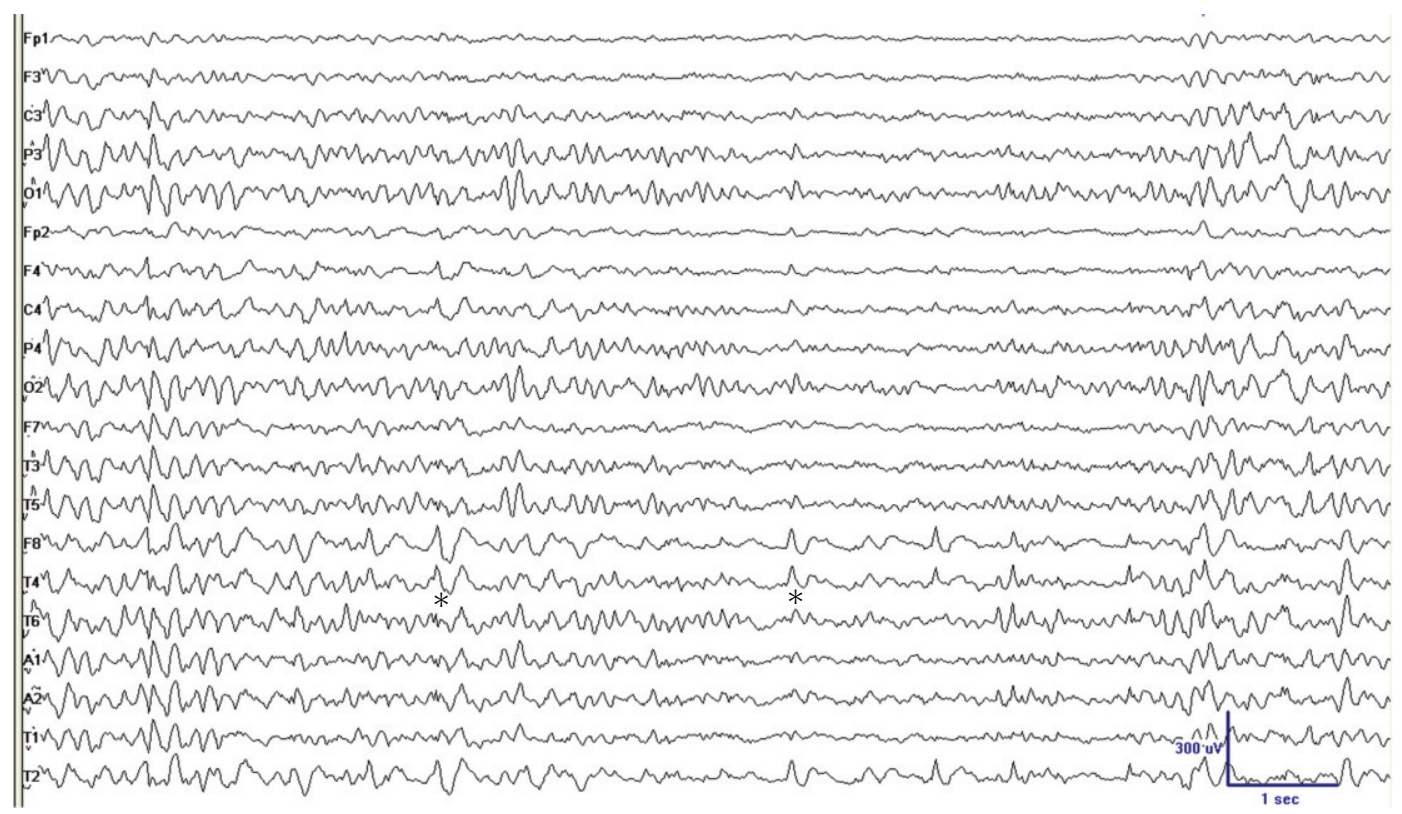

(b)

FIGURE 1: Typical EEG in a patient with right mTLE showing theta-delta activity over the right temporal region and right temporal spikes $(*)$ with phase reversal across F8 and T4 electrodes (The same epoch reformatted (a) bipolar montage and (b) common average referential montage; $\mathrm{LFF}=1 \mathrm{~Hz} ; \mathrm{HFF}=70 \mathrm{~Hz})$.

earlier discernible on SP than scalp thus increasing seizure lateralization $(5.4-7 \%$ increased yield) [24, 25]. Dipole localization techniques with SP electrodes also help with accurate source localization [26]. Blindly placed sphenoidal electrodes often do not lie below anatomical foramen ovale and may account for reduced efficiency [27]. Significant anatomical migration of the SP electrodes is also inevitable with prolonged recordings [28].

\section{Interictal EEG Findings in TLE}

Preoperative interictal EEG abnormalities commonly observed in TLE are focal arrhythmic slowing (either theta or delta) and focal IEDs that are often restricted to the anterior temporal areas (Figures 1(a) and 1(b)). In majority, these abnormalities correlate well with SOZ and the structural abnormalities seen on magnetic resonance imaging (MRI) (illustrative case 1). In TLE, single or 


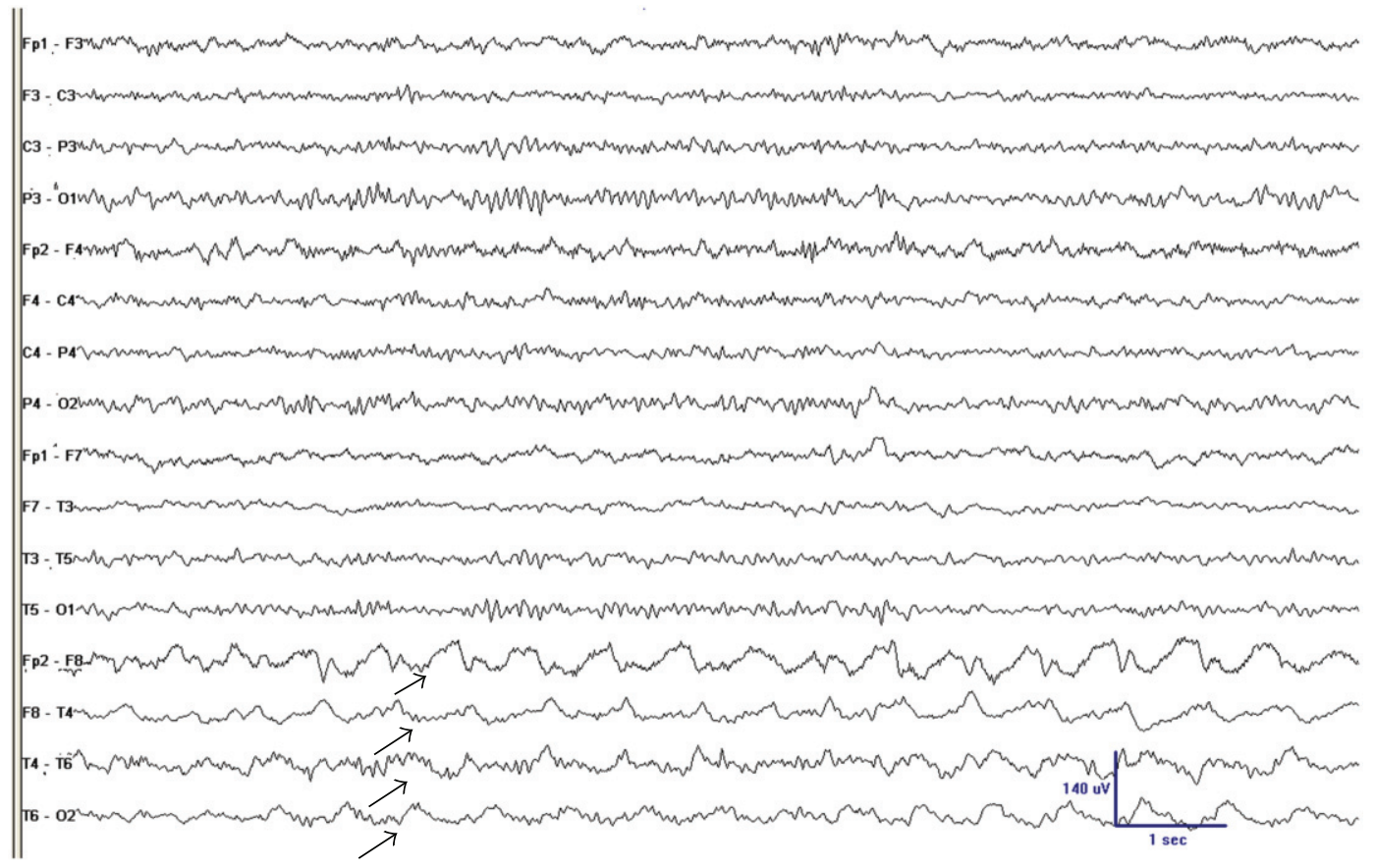

FIGURE 2: Bipolar anterior to posterior montage with 10-20 system showing 1-1.5 Hz temporal intermittent rhythmic delta activity (TIRDA) over the right temporal region (arrows) with maximal amplitude over anterior temporal region. Patient was a 42-year-old man with refractory CPS, right MTS, right temporal IEDs, and right temporal CPS (VEEG recordings). He was rendered seizure-free with right TLY and remained seizure-free at 2-year followup (LFF $1 \mathrm{~Hz}-\mathrm{HFF} 70 \mathrm{~Hz}$ ).

serial outpatient EEGs demonstrate strong correlation of interictal abnormalities with areas of surgical resection and postoperative seizure outcomes (90\% for IEDs and $82 \%$ for focal slowing) $[29,30]$. Such strong correlations may obviate the need for mandatory ictal recordings during presurgical workup in patients with unilateral hippocampal atrophy (HA) and congruent clinical and neuropsychological data [31]. However, ictal recording becomes essential to rule out the possibility of concurrent psychogenic nonepileptic seizures (PNESs) [32]. Moreover, bilateral TLE, coexisting extratemporal epilepsy, or generalized epilepsy may not be appreciated in routine outpatient scalp EEGs.

IEDs and clinical semiology aid to differentiate between mesial TLE (mTLE) and lateral or neocortical TLE (nTLE) [33]. The IEDs remain lateralized to the temporal regions in both syndromes (illustrative cases 1 and 2). In mTLE, IEDs are dominant over the anterior mesial temporal areas (T1/2, A1/2, F7/8, and T3/4), while patients with nTLE tend to have more lateral and posterior temporal IEDs (T5/6). While mesial temporal IEDs can infrequently occur in nTLE, converse is unlikely, that is, mTLE patients usually do not have neocortical IEDs. In TLE associated with MTS, IEDs tend to be more localized to the anterior temporal regions, while in the TLE associated with tumors, there is an increased tendency for bilateral expression of IEDs [34]. Typical anterior temporal spikes can also be seen in extratemporal epilepsy (a.k.a. temporal plus syndrome) [35, 36] (illustrative cases 7, 8).

Approximately 30 percent patients with unilateral TLE on other evaluation parameters show bitemporal IEDs [37,
38]. Many of these patients with refractory epilepsy do well with epilepsy surgery. However, greater degree of bilateral IEDs trends towards lesser postoperative seizure outcomes [39]. Chung et al. [40] in an invasive depth study of patients with unilateral SOZ demonstrated that greater than 90 percent lateralization of IEDS resulted in good surgical outcome in more than 90 percent of patients. Less than 90 percent laterality of IEDs resulted only in 50 percent of patients having good surgical outcome. Additionally, SOZ also predicts surgical outcomes in patients with bilateral temporal IEDs. Bilateral SOZ and bilateral IEDs have very poor outcome with surgery (only $12 \%$ seizure-free), while those with bilateral IEDs but unilateral SOZ have favorable outcome (40 to 56 percent) [41]. Repeated video EEG recordings may be at times necessary to demonstrate consistency of ictal laterality in TLE with bilateral IEDs [42]. Other findings such as MRI abnormality, hippocampal sclerosis (HS), neuropsychology, and clinical details help to determine the degree of laterality and the surgical outcome $[42,43]$. Interestingly, in many with bilateral IEDs and good postoperative outcome followup, EEGs may show reduction or disappearance of contralateral spikes thus supporting the "seizure-induced epileptogenesis" hypotheses for contralateral IEDs [44] (case 6). Temporal IEDs also predict ictal scalp pattern. Lateralized ictal patterns are more common with unilateral temporal IEDs than bilateral [45]. The presence of bisynchronous IEDs in unilateral TLE is predictive of higher incidence for generalized seizures, but such patients still have favorable surgical outcome [46]. 


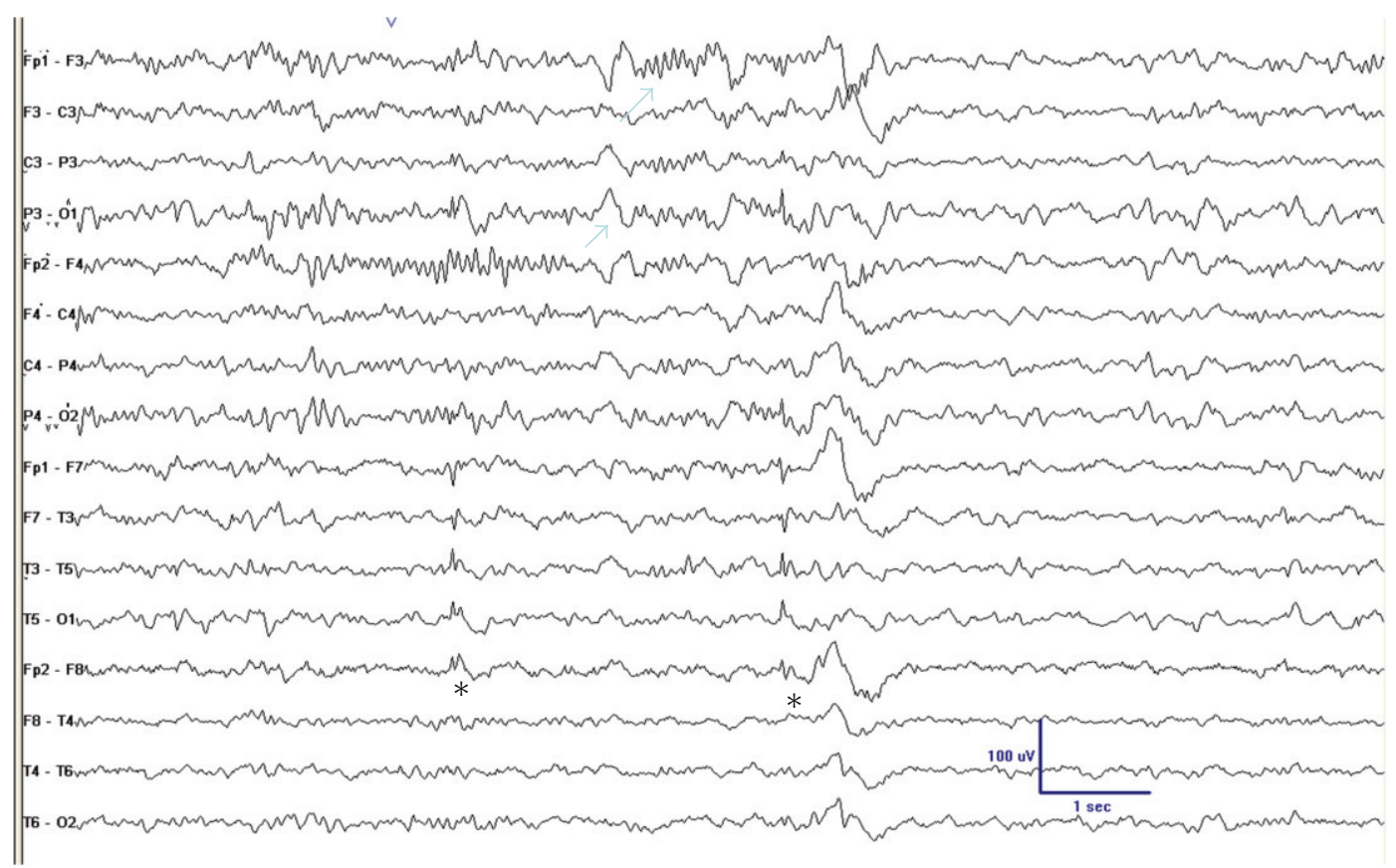

(a)

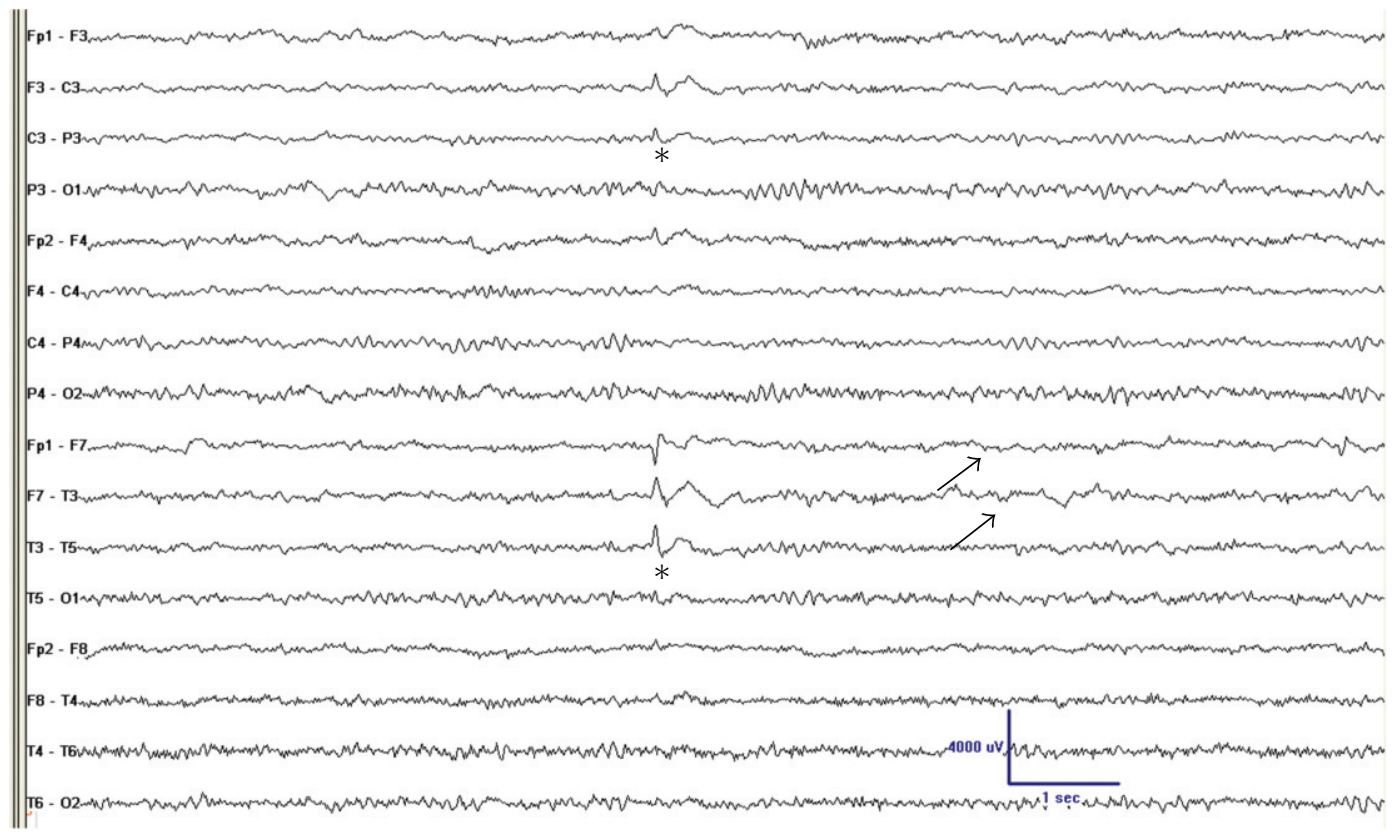

(b)

FIGURE 3: EEG in a 21-year-old man with complex partial seizures and normal MRI showing small sharp spikes (a) in sleep ( $*$ ) and left temporal spike $(*)$ and slowing (arrows) during wakefulness (b). Note the very frequent occurrence of small sharp spikes and its localization to the left temporal lobe where IEDs and slowing occurred $(\mathrm{LFF}=1 \mathrm{~Hz} ; \mathrm{HFF}=70 \mathrm{~Hz})$.

Temporal "plus" epilepsies are characterized by seizures involving a complex epileptogenic network of the temporal lobe and the neighboring structures such as the orbitofrontal cortex, the insula, the frontal and parietal operculum, and the temporo-parieto-occipital junction [47-49]. Neither temporal IEDs nor temporal SOZ effectively rules out "temporal lobe plus" epilepsies (Figure 17). These patients are often recognized by a combination of clinical, imaging, and EEG findings. The IEDs are often precentral and bilateral in these patients. They often need invasive monitoring to localize the SOZ. Insular seizures due to dense temporolimbic connections have clinical manifestations very similar to TLE 


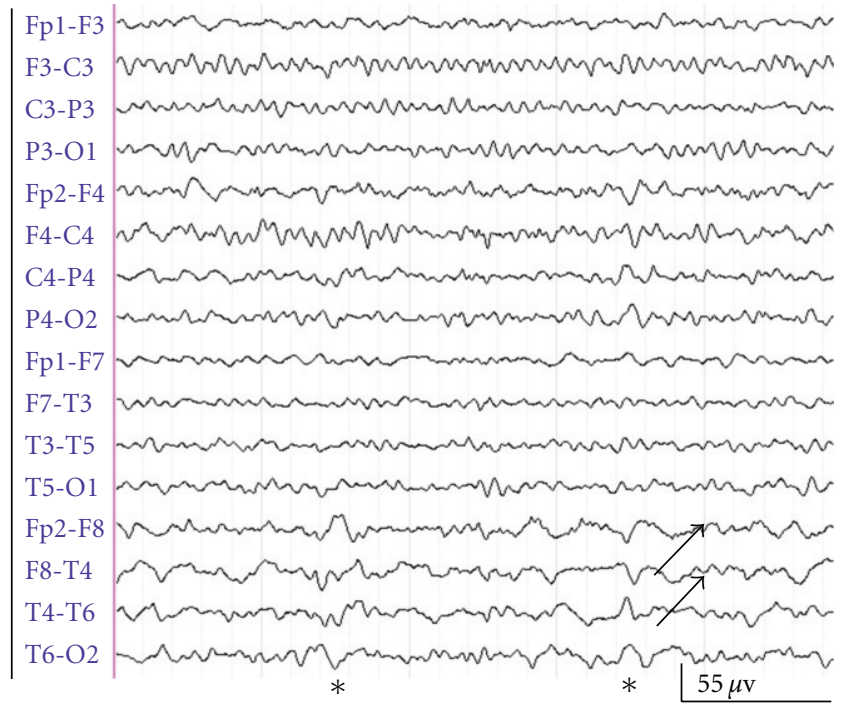

(a)

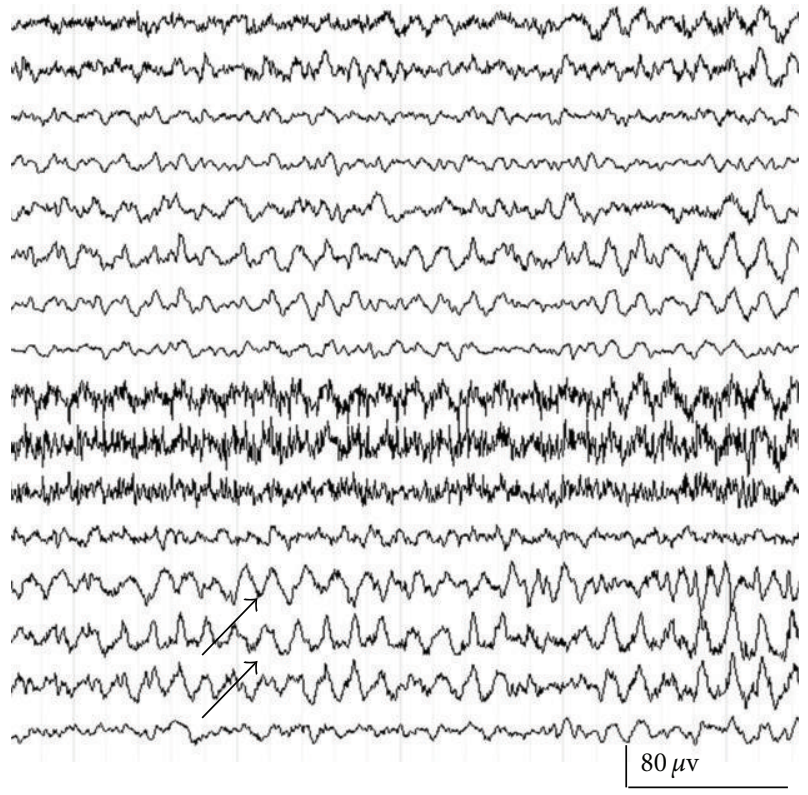

(b)

Figure 4: (Case 1) (a) Theta-delta slowing (arrows) and sharp waves $(*)$ over the right temporal region (phase reversal across T4) in anterior-posterior bipolar montage in right MTLE. (b) Ebersole ictal pattern type I involving the right temporal region (arrows) in one of the complex partial seizures recorded during continuous video $(\mathrm{LFF}=1 \mathrm{~Hz} ; \mathrm{HFF}=70 \mathrm{~Hz}$ ).

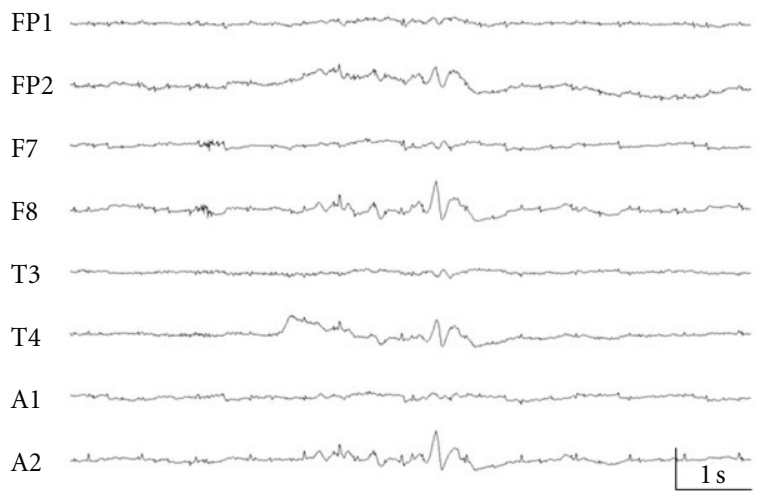

Common average referential recording: Sensitivity $=10 \mathrm{uv} / \mathrm{mm}, \mathrm{LFF}=0.1 \mathrm{~Hz}$, HFF off

Figure 5: (Case 2) A typical right temporal IED involving F8, A2, and T4 during sleep $(\mathrm{LFF}=0.1 \mathrm{~Hz} ; \mathrm{HFF}=70 \mathrm{~Hz})$ in a patient with refractory CPS secondary to right temporal neocortical cavernous hemangioma.

[50-53]. Such seizures are characterized by a sensation of constriction in the throat, paresthesias, or warmth feeling over the perioral region or large body territories, followed by focal sensory-motor manifestations. Positron emission tomography (PET) and ictal single-photon emission computed tomography (SPECT) studies may help identify few of these patients with nonlesional insular seizures [54] (illustrative case 7).

The electrical dipole nature of the IEDs has a prognostic value. A relatively localized negativity with steep

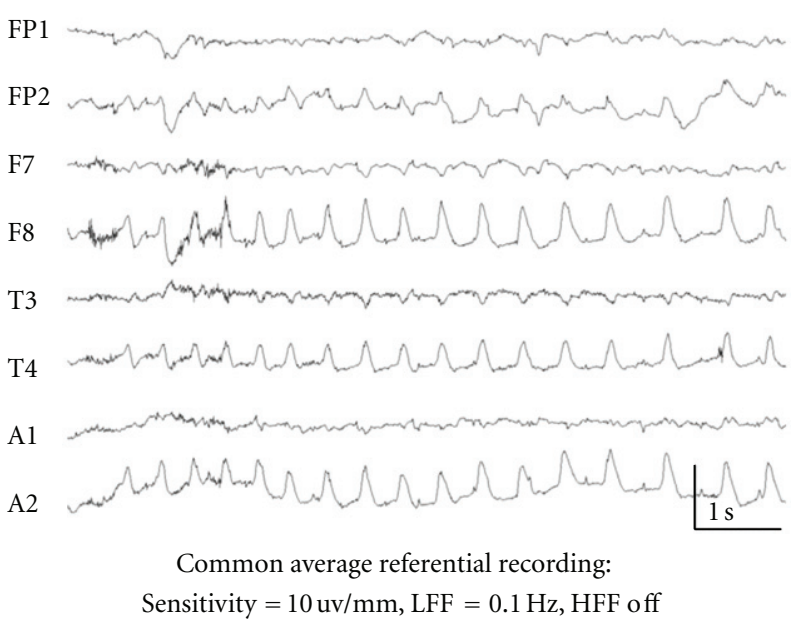

Figure 6: (Case 2) Ictal changes as right temporal seizure onset (F8, A2, and T4) with minimal involvement of the right frontopolar (FP2) region (type 2 ictal rhythm).

voltage gradient at the anterior temporal electrodes or sphenoidal electrodes with widespread vertex positivity termed as "Ebersole type 1 source" localizes the abnormality to mesiobasal temporal lobe (illustrative case 1 ). These patients are likely to have a very good surgical outcome. IEDs with relatively broad frontal-basal negativity that may cross the midline, gradual voltage gradient, and poor electropositivity ("Ebersole type 2 source") indicate either nTLE or frontal originating spikes $[55,56]$ (illustrative case 2 ). 


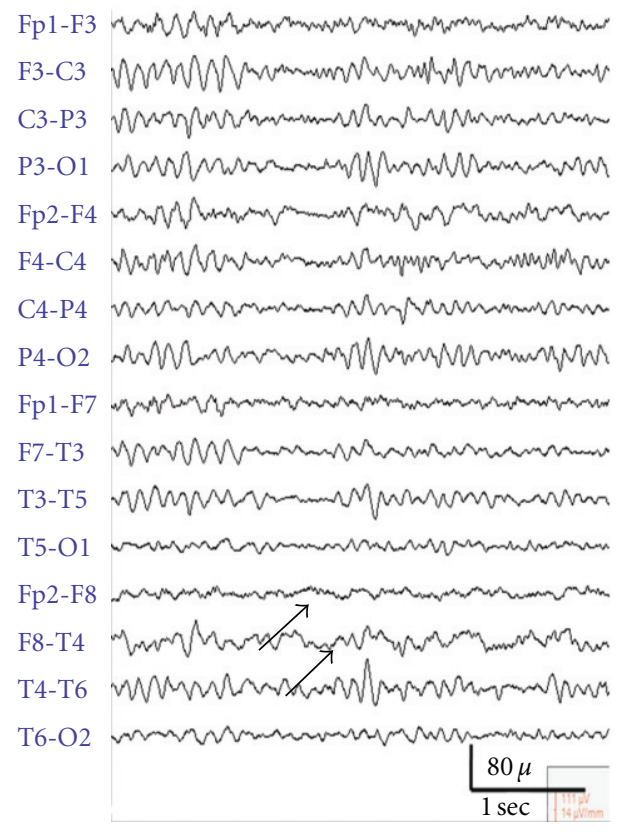

(a)

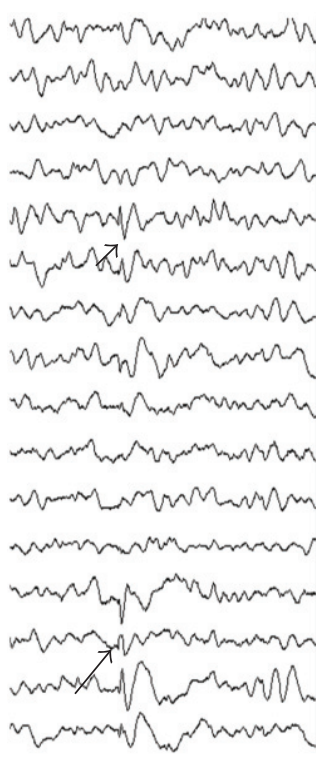

(b)

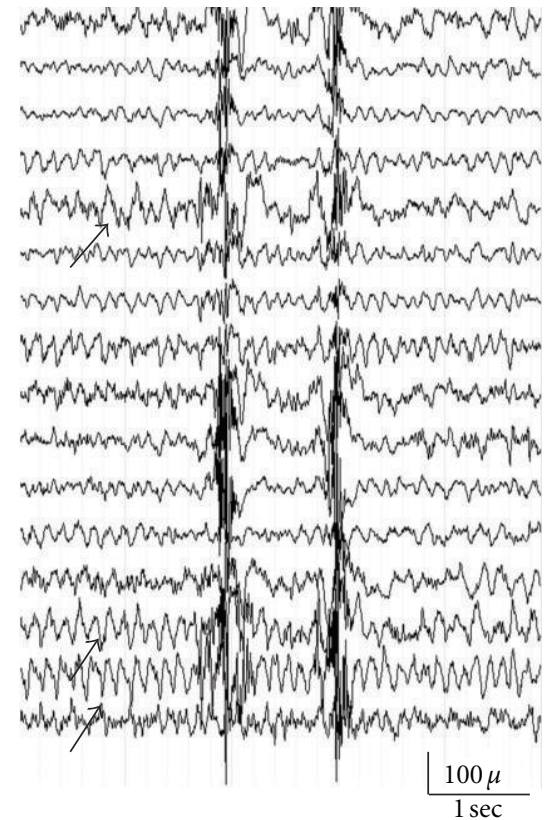

(c)

Figure 7: (Case 3) Interictal EEG in a patient with medically refractory complex partial seizures and right posterior temporal lesion on MRI (Figure 8) shows mild right anterior temporal slowing ((a) arrows) and rare right anterior temporal spikes with minimal spread to the frontopolar region ((b) arrows). Ictal rhythm during a complex partial seizure shows right temporal regionalized semirhythmic theta activity ((c) arrows).

Frequent IEDs (i.e., 60 spikes/hour in one EEG recording) are associated with less than desirable outcome after temporal lobectomy (TLY) [57]. This supports the "mouse model hypothesis" that IEDs are inhibitory phenomena, and they help to control seizures [58]. A small subset of patients with TLE have infrequent or absent IEDs (cases $2,3)$. Such "oligospikers" (i.e., IEDs < one in an hour on several scalp EEG recordings) tend to have a good ictal localization and excellent surgical outcomes similar to other TLE patients. Oligospikes in TLE often correlate with later age of seizure onset, low seizure frequency, lesser tendency for status epilepticus (SE), or hippocampal atrophy (HA). This subset likely represents milder degree of MTS without differences in etiological factors [59]. Absence of IEDs may suggest extratemporal seizures, and such patients may need extra care during presurgical workup [60].

Small sharp spikes (SSS) or benign epileptiform transients of sleep (BETS) are benign epileptiform variants common in adolescents and young adults. These are typically monophasic or diphasic discharges, without aftercoming slow wave, having widespread distribution and are often most prominent over the anterior temporal and frontal regions. They occur sporadically and independently over both hemispheres and are often seen during stages 1 or 2 of NREM sleep. Pathological SSS or unilateral SSS (often with theta) may be linked to complex partial seizures tend to occur in deeper stages of sleep, wakefulness, in couplets and often localize to one of the anterior temporal lobes from where the seizure arises [61] (Figure 3).

\section{Temporal Intermittent Rhythmic Delta Activity (TIRDA)}

In TLE, interictal EEG (often in drowsiness or light sleep) shows a rhythmic sinusoidal $1-4 \mathrm{~Hz}$ delta activity that remains localized to the temporal lobes (Figure 2). TIRDA has high correlation with anterior temporal IEDS, SOZ, mesial, and mesiolateral TLE, particularly in patients with MTS. Lateralized TIPDA is more common in patients with extratemporal epilepsy (about 20\%) [62, 63].

\section{Ictal Rhythms in TLE}

In majority (approximately 90\%) with unilateral TLE (unilateral MRI abnormality and IEDs), the ictal lateralization corresponds to interictal IEDs and slowing. Ictal rhythms can be variable even within the same patient. Lateralization at onset can be observed in only a third of unilateral TLE $[30,64]$. Ictal EEG may not aid in differentiating the anterior from posterior lateral TLE [65]. Ebersole et al. classified the ictal rhythms in TLE into three types [66]. Typical ictal surface EEG with high interrater concordance consists of a rhythmic 5 to $9 \mathrm{~Hz}$ theta activity that slowly evolves and remains localized to the temporal or subtemporal regions which is termed type 1 ictal rhythm (illustrative case 1 ). This pattern is most specific for hippocampal seizures. A lower frequency of 2 to $5 \mathrm{~Hz}$ irregular ictal rhythm with widespread temporal distribution is termed as "type 2 rhythm" and is 


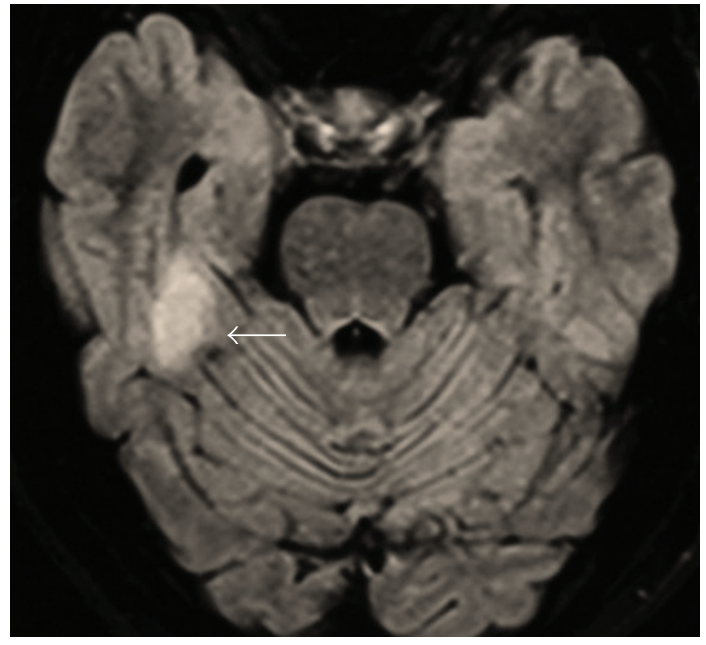

(a)

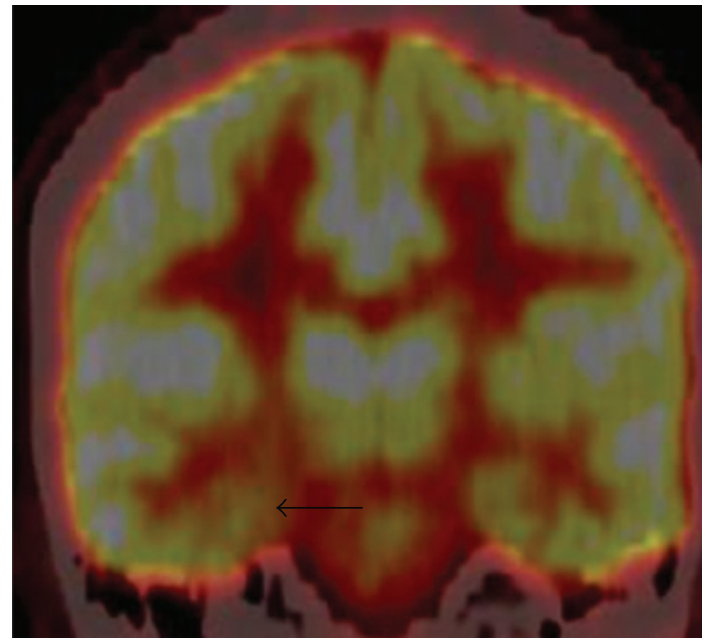

(c)

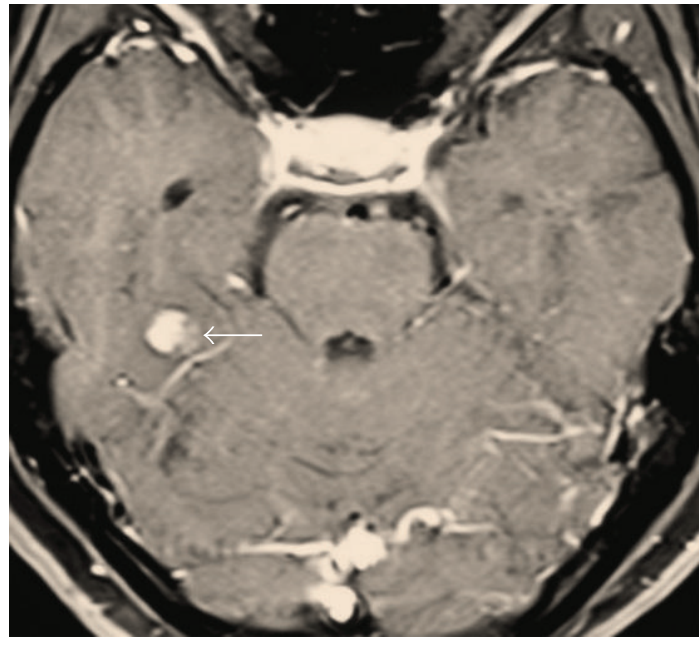

(b)

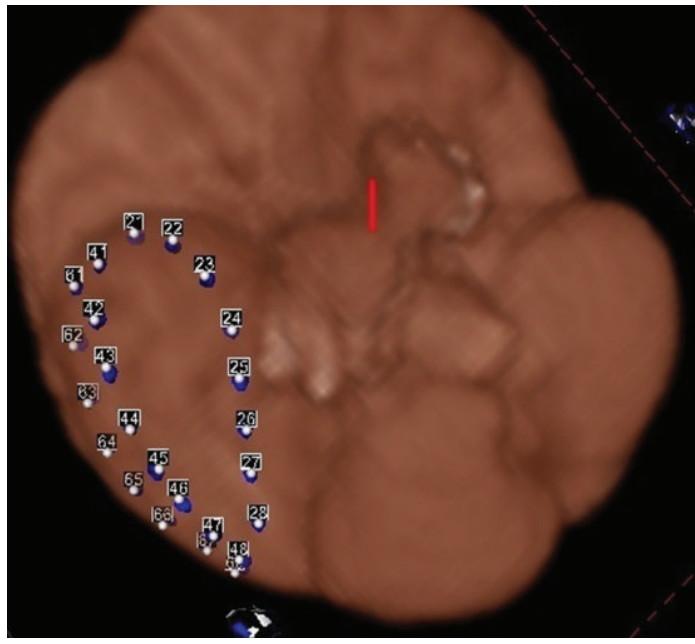

(d)

FIgURE 8: (Case 3) MRI of the brain ((a) FLAIR and (b) contrast) shows a right posterior temporal lesion that is enhanced with contrast injection. FDG-PET (c) scan shows mild right temporal hypometabolism. (d) Coregistered image of the location of the subdural lines during invasive monitoring (8 contacts and three strips; the image was obtained with Atamai Epilepsy Viewer software [6]).

often associated with neocortical seizures [67] (illustrative case 2). Diffuse ictal EEG changes or attenuation without clear lateralization (type 3 rhythm) can be seen both in hippocampal and temporal neocortical seizures.

\section{Comparison of Surface EEG with Invasive Recordings}

Correlating studies comparing surface EEG with simultaneous subdural electrodes (SEs) and depth electrodes (DEs) demonstrate that most subclinical electrical seizures confined to the hippocampus do not result in surface EEG changes (illustrative case 5: subdural recordings). When the seizure spreads from mesial temporal to the inferolateral temporal structures, type I surface rhythm is observed.
Type 2 rhythms are often neocortical seizures starting as fast activity of 20 to $40 \mathrm{~Hz}$ on subdural electrodes that are either not detectable on surface EEG or are seen as "attenuation patterns" followed by asynchronous theta-delta activity over the temporal regions. Type 3 ictal rhythm occurs when seizures are confined to the hippocampus or spreads rapidly to the contralateral hippocampus, where there is little synchronisation of the electrical activity over the inferior lateral temporal structures for expression on to the surface EEG.

Start-stop-start is an ictal phenomenon observed in TLE when initial ictal pattern is followed by complete cessation often reverting back to interictal EEG and then again reappearance of ictal potentials. In about a third, the restart may occur at a different anatomical location than the initial start, thus a potential pit fall of ictal localization [68]. 


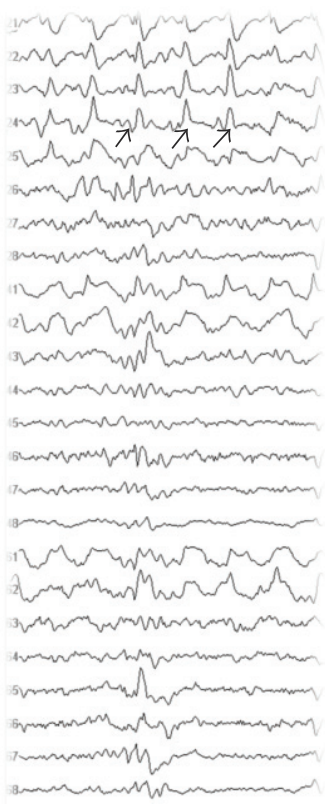

(a)

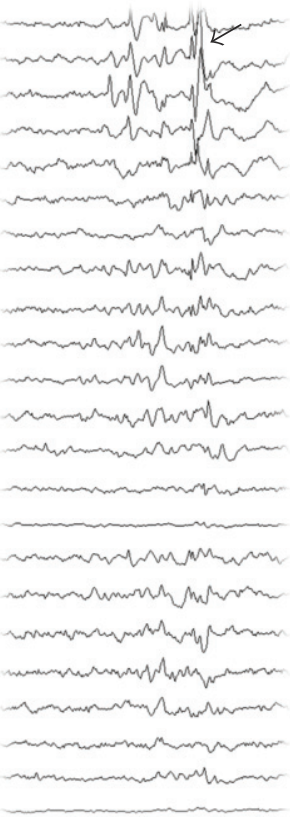

(b)

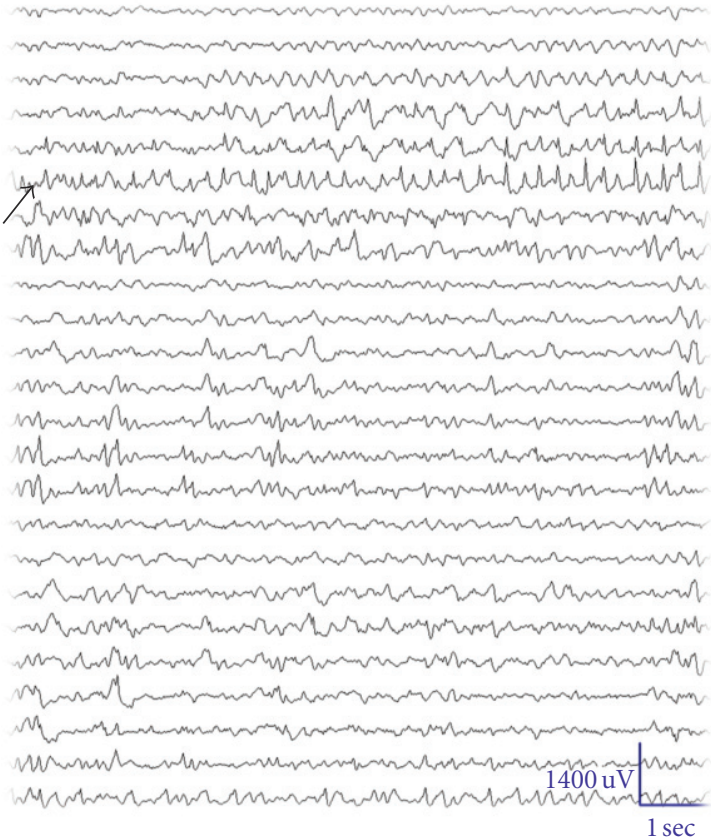

(c)

Figure 9: (Case 3) ((a) and (b)) Subdural recordings show temporal polar and anterior mesial temporal region IEDs (arrows, (a) rhythmic spikes, (b) sporadic). (c) An electrographic seizure involving the parahippocampal structures (arrow) without clinical manifestations.

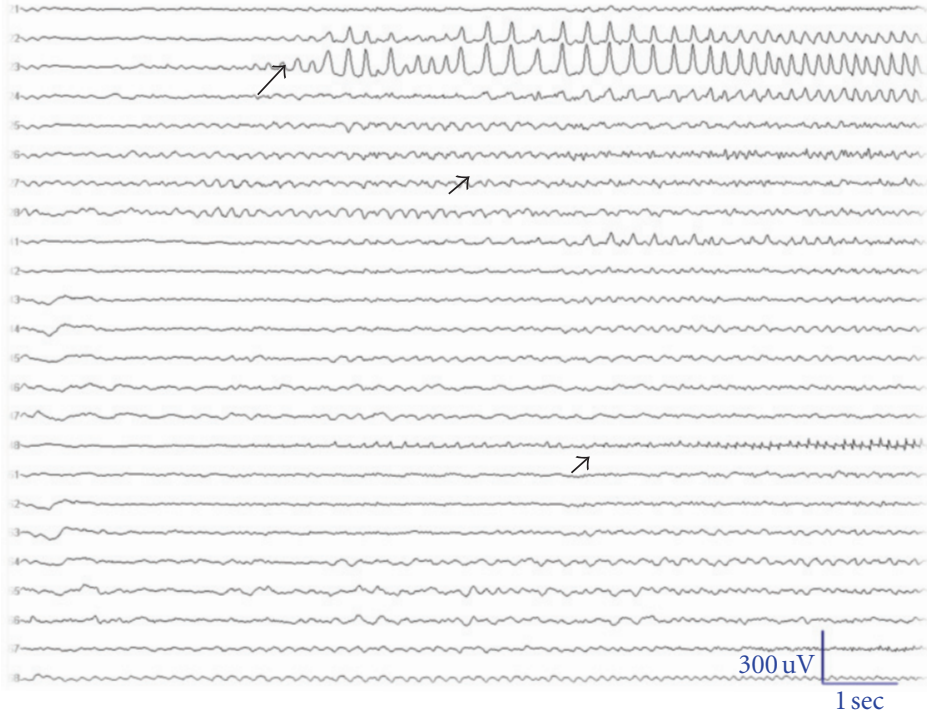

(a)

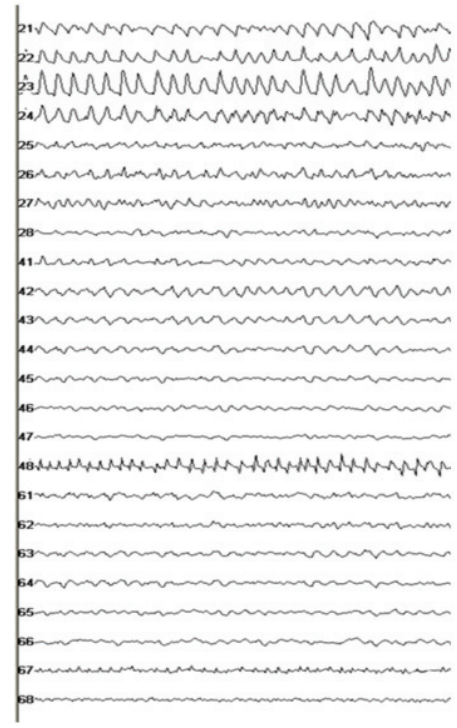

(b)

Figure 10: (Case 3) Ictal EEG shows $3 \mathrm{~Hz}$ evolving rhythm in the temporal polar and mesial temporal regions (long arrow) with faster rhythm posteriorly (short arrows) within 2 to 3 seconds (Note that (a) and (b) are contiguous time frame).

\section{Ictal Propagation Patterns}

Temporal lobe seizures often use indirect pathway for propagation into the contralateral temporal lobe more than direct hippocampal commissures [69]. The orbitofrontal cortex is strongly influenced by mesial temporal ictal activity [70]. In majority, the propagation is to ipsilateral frontal lobe, the contralateral frontal lobe, and then to the contralateral temporal lobe.

Early propagation of seizures (less than 10 seconds) suggests more widespread hyperexcitability and greater probability of bilateral temporal epileptogenicity and tends to occur in patients other than pure MTS [39, 71, 72]. Best surgical benefits can be expected in those patients with 


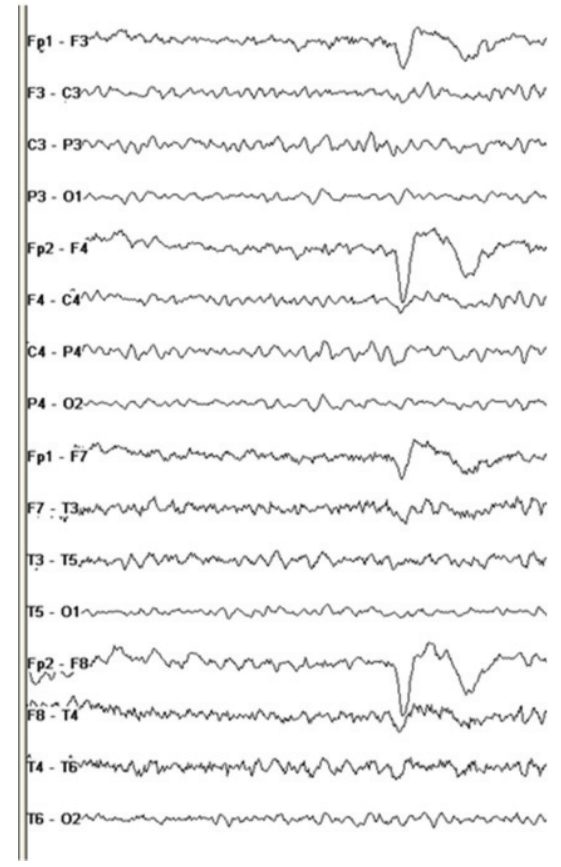

(a)

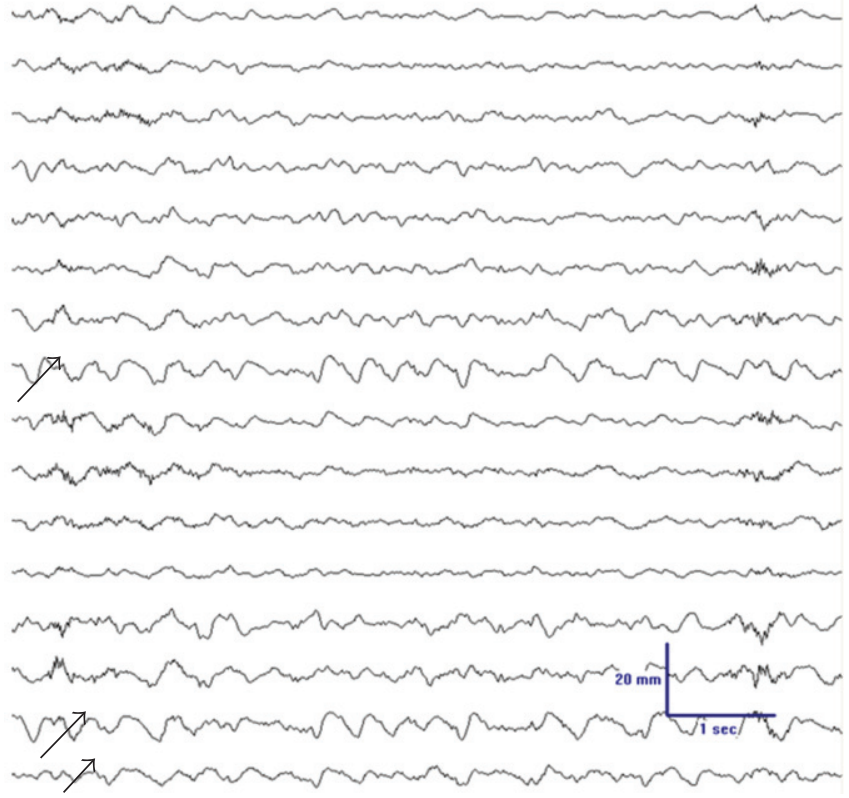

(b)

Figure 11: (Case 4) (a) Normal EEG in a 20-year-old man with refractory complex partial seizures and right posterior temporal enhancing lesion. (b) Ictal EEG about 30 seconds into one of the typical complex partial seizures shows arrhythmic theta-delta activity over the right temporal-occipital regions. No clear lateralizing ictal EEG changes were discernible early in the seizure (not shown here).

regionalized ictal EEG activity without contralateral spread and ipsilateral interictal abnormalities.

\section{Invasive Monitoring: Depth, Subdural Lines, or Both}

Invasive monitoring can be safely performed either with SE or DE when noninvasive data are discordant $[73,74]$. The most important step prior to embarking upon invasive recording is an "unbiased hypothesis." Indications for invasive recordings in TLE are either bitemporal epilepsy or temporal plus syndromes [75].

Hippocampal DEs (6-8 contacts) are placed stereotactically along the long axis of hippocampus to amygdala through a small occipital burr hole. These are particularly useful in mTLE of uncertain lateralization. However, DE recordings alone do not differentiate mesial from neocortical TLE. DEs are not useful in isolation for "temporal plus" syndromes. A combination of SEs or grids with DEs tailored to an individual patient becomes essential. This is either performed through multiple subdural electrodes (for further details on the placement of invasive EEG electrodes, see Steven et al. [76]) or by a combination of a subdural grid covering the lateral temporal lobe, while the mesial structures are covered by two or three DEs ( 4 contacts) perpendicular through the middle temporal gyrus and the overlying grid $[77,78]$. Both of these techniques are equally effective. In TLE, there is a high degree of concordance between the SE and DE recordings particularly if the electrode placement is mesial to the collateral sulcus and is recording from the surface of parahippocampal gyrus [79] (illustrative cases 3 and 5). SE/grids also provide an opportunity for functional language localization by stimulation.

In general, most of the seizures recorded by SE arise from the same lobe showing predominant IEDs and seizures on scalp EEGs [80]. On occasions, auras and subclinical seizures detected by DE recordings may not be evident on SE [81]. Presence of periodic IEDs prior to the seizure onset in mesial temporal lobe structures is often specific for hippocampal onset seizures and correlates well to reduced CA1 cell counts. Temporal neocortical seizures at onset have significantly faster frequencies $(20$ to $40 \mathrm{~Hz}$ ) in contrast to the hippocampal seizures that have slower frequencies (13 to $20 \mathrm{~Hz}$ ). TLE associated with MTS is more likely to have higher seizure onset frequency than mTLE not associated with MTS (illustrative cases 3 and 5).

Two common seizure patterns in temporal lobe seizures are hypersynchronous rhythmic high-amplitude activity (HYA) and low-voltage fast activity (LVFA) (illustrative cases 3 and 5). HYA is likely to represent more focal onset and lesser rate of spread to contralateral mesial temporal structures and is associated with more marked neuronal loss in the hippocampi than the LVFA that tends to be more regionalized and neocortical in nature involving both hippocampal and extrahippocampal networks [82-85]. Seizures with LVFA or rhythmic sinusoidal ictal patterns are associated with better outcomes after surgery [86].

Following seizure onset and initial recruitment of the surrounding area, the ictal rhythm propagates variably. The 


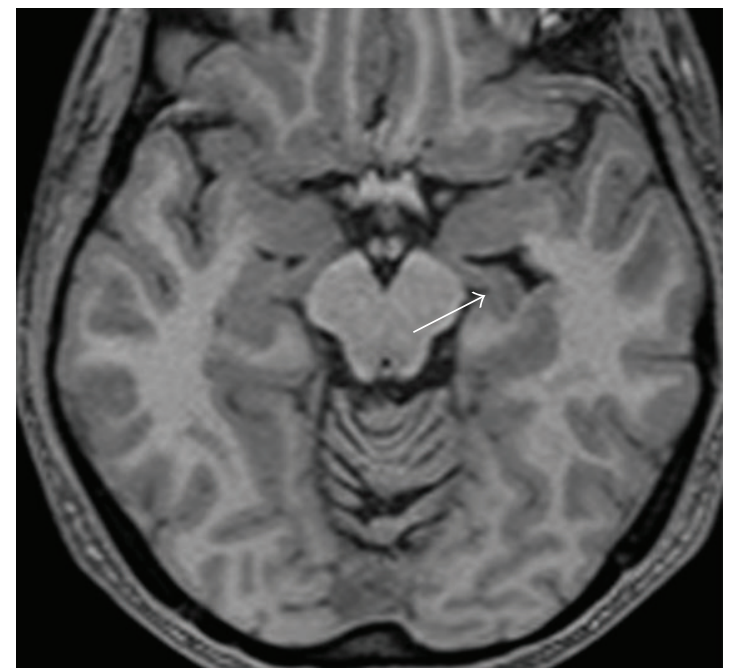

(a)

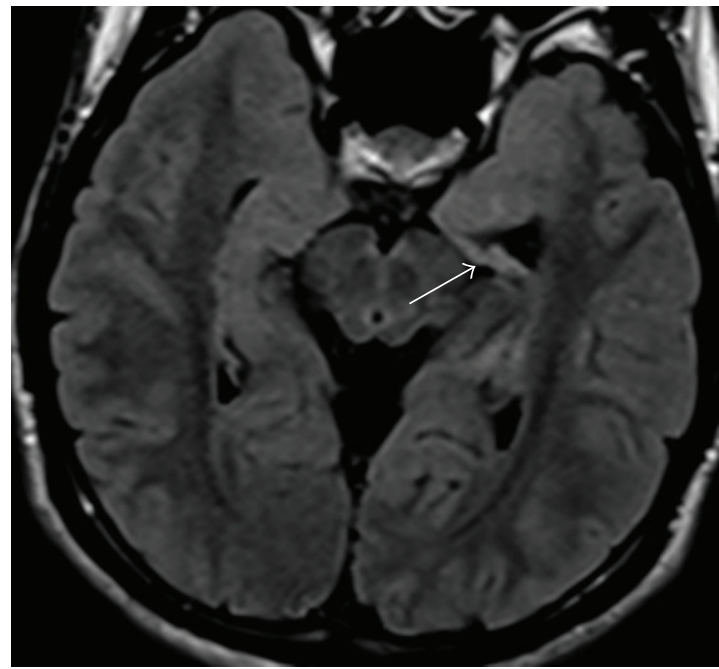

(b)

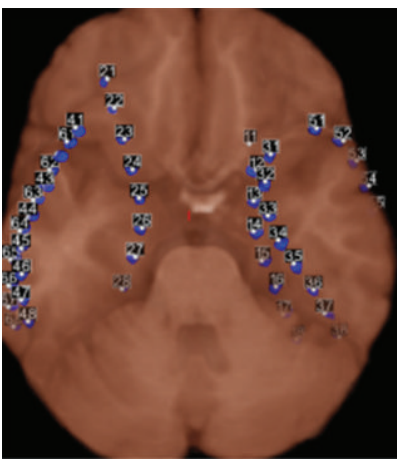

(c)

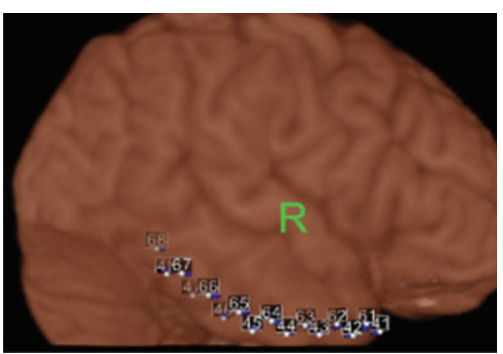

(d)

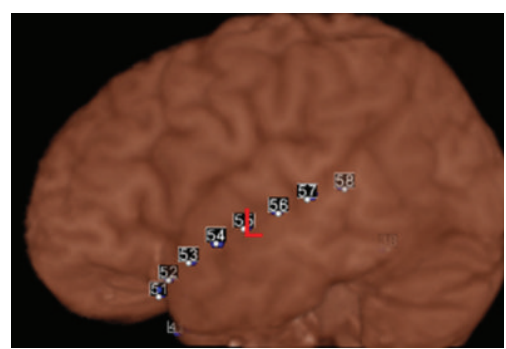

(e)

FIGURE 12: (Case 5) MRI of the brain in a patient with refractory complex partial seizures shows severe left MTS (upper). Atami Epilepsy Viewer image of bilateral subdural lines placed for invasive monitoring (lower).

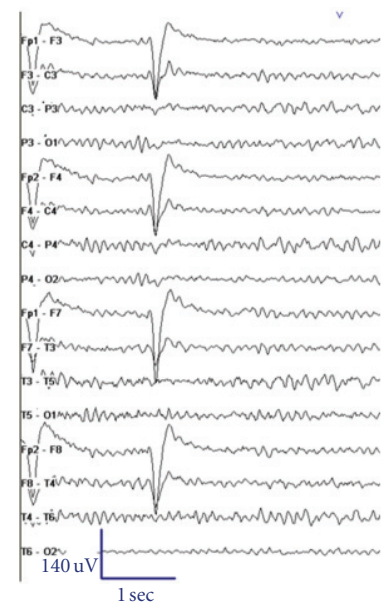

(a)

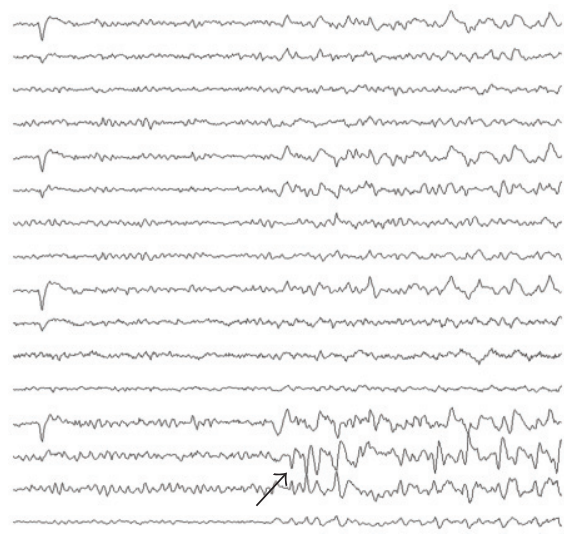

(b)

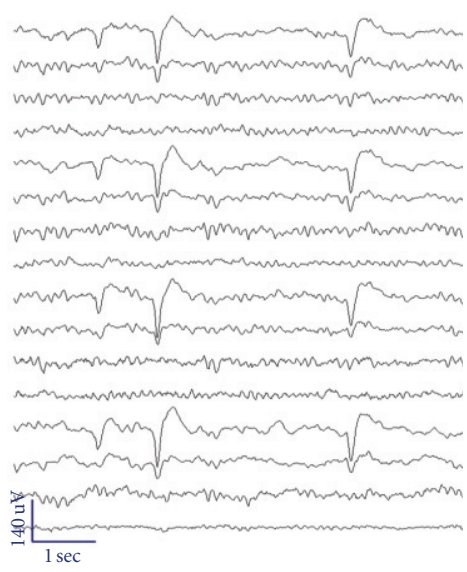

(c)

FIgure 13: (Case 5) Normal EEG (a) in a patient with refractory complex partial seizures and left mesial temporal sclerosis. Ictal EEG at onset during one of the CPSs (b) (arrows) shows semirhythmic theta onset over the right temporal regions (arrow). Postictal EEG (c) shows right temporal rhythmic delta activity (arrow). 


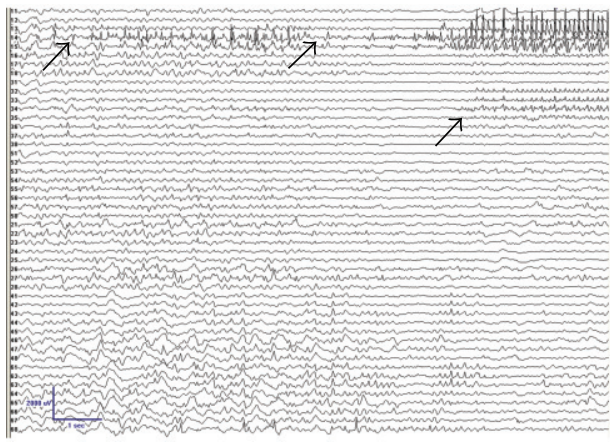

(a)

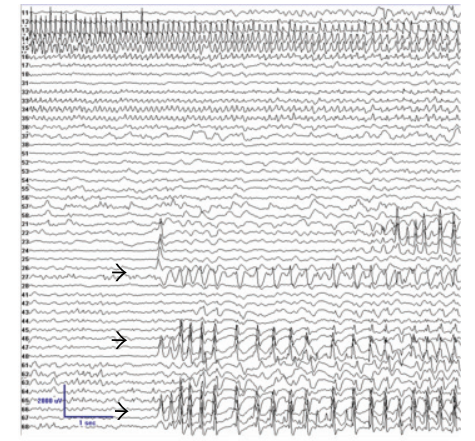

(b)

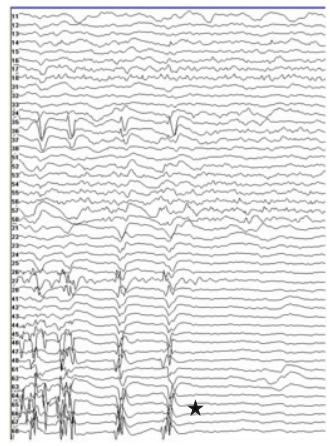

(c)

FIGURE 14: Subdural ictal EEG shows rhythmic spikes arising from the left hippocampal area followed by localised low-voltage fast activity and then sequential spikes spreading to the left subtemporal area (a) (arrows) but not to the left temporal neocortex. (b) About 12 seconds into the seizure, it spreads to the right temporal regions (both mesial and neocortical) (arrow heads) with continued activity in the left temporal region with offset in right temporal lobe (c). Prominent postictal slowing over the right temporal region is seen (c).

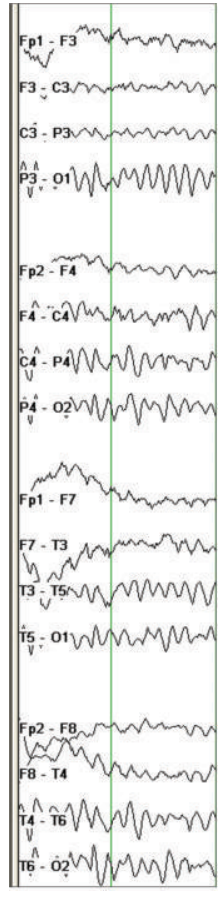

(a)

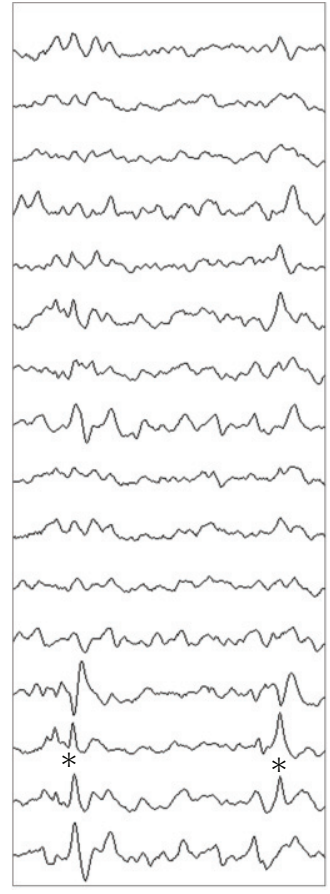

(b)

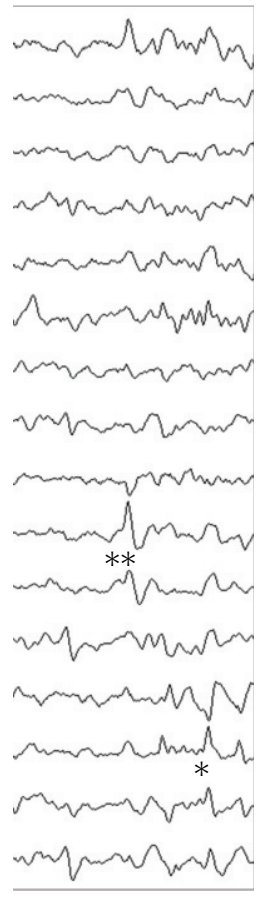

(c)
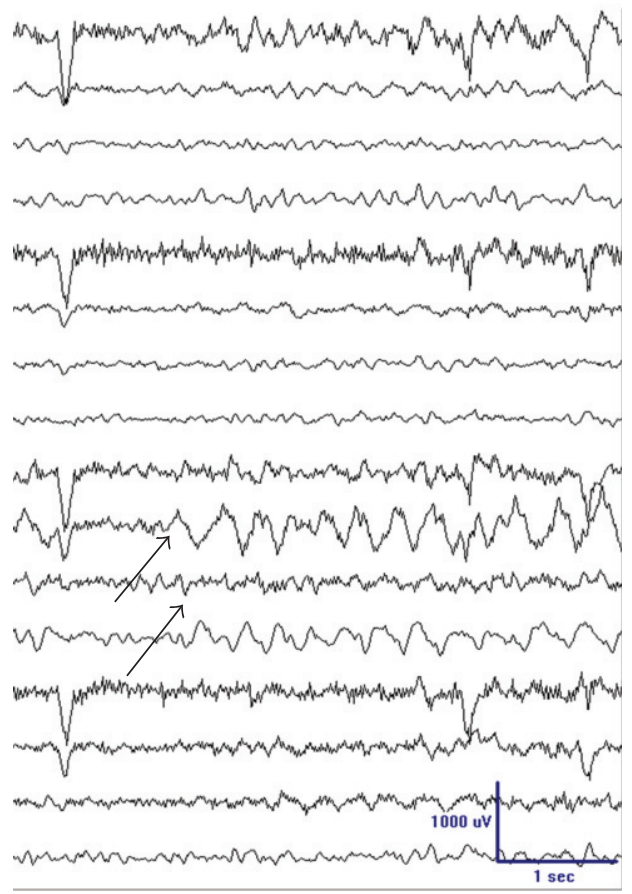

(d)

FIGURE 15: (Case 6) EEG in a 38-year-old woman with refractory complex partial seizures and left MTS. (a) Background EEG that showed mild independent bilateral temporal and generalized slowing; (b) right anterior temporal spikes (*); (c) left anterior temporal spikes $(* *)$ (right temporal IEDs to left temporal IEDs $70: 30$ ) in sleep; (d) left temporal type 2 ictal rhythm as rhythmic delta activity in the left temporal regions (arrows) during one of her typical complex partial seizures (seizure onset zone). A total of 16 seizures were captured from the left temporal region. She underwent a left ATL and was seizure-free at last followup 12 months and had a marked reduction in the right temporal IEDs.

spread can be to the ipsilateral temporal lobe, contralateral mesial temporal, or temporal neocortex [87]. Long interhemispheric propagation times are associated with good surgical outcomes in mTLE. Time to propagation of the seizure to the contralateral hippocampus is directly proportional to cell loss in the Cornu Ammonis (CA) subfield 4 (CA4, also known as the hilar region of the dentate gyrus), suggesting a role for CA4 in this process [88].

\section{Wasted Hippocampal Syndrome}

In rare instances, one may come across patients with severe unilateral HA with contralateral ictal onset of seizures. These patients are often referred to have "wasted hippocampal syndrome" (illustrative case 5) [89]. In vast majority of patients, invasive recordings show seizures arising from the atrophic side, and they have very good seizure outcomes with 


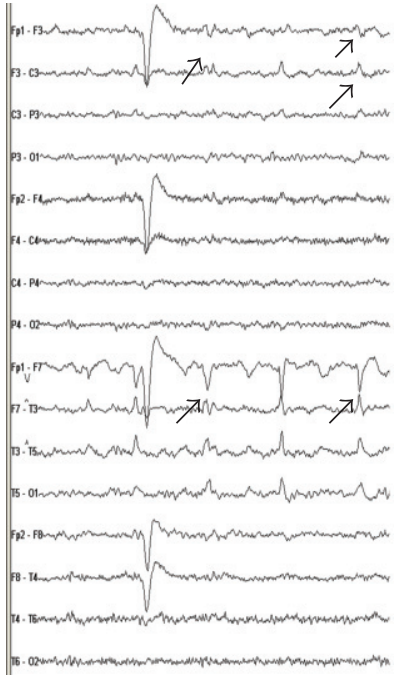

(a)

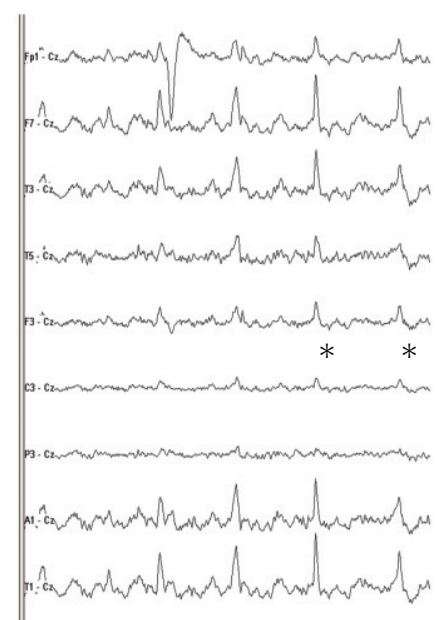

(b)

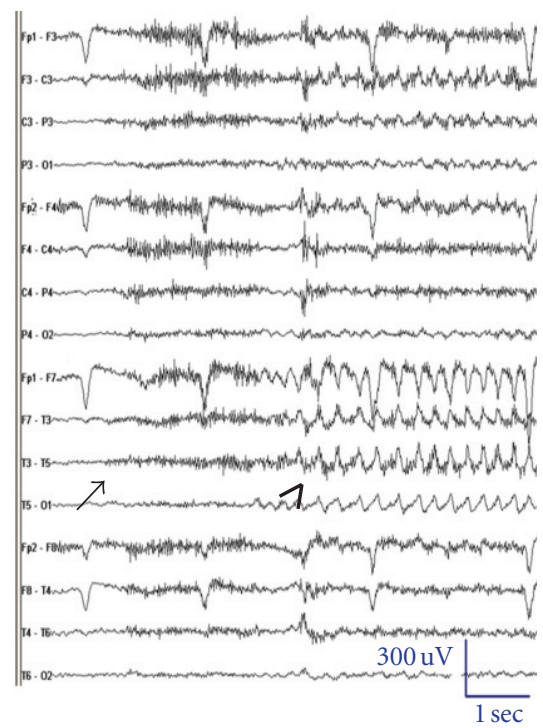

(c)

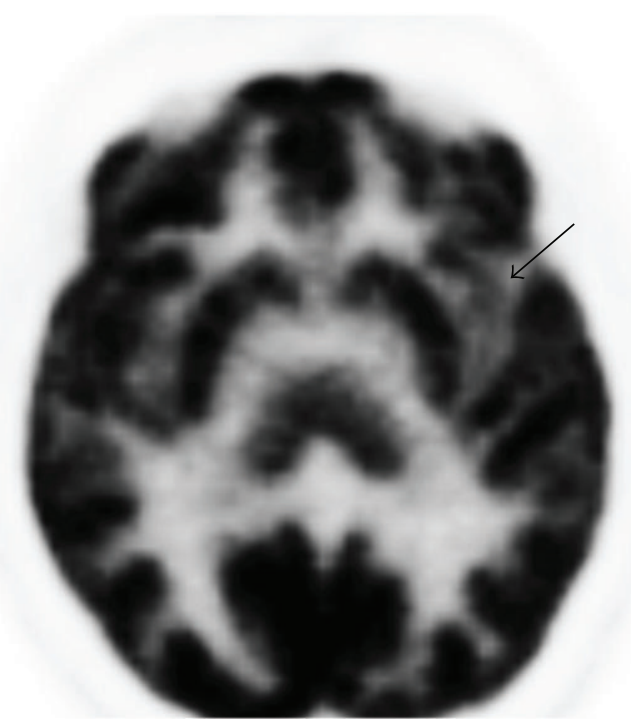

(d)

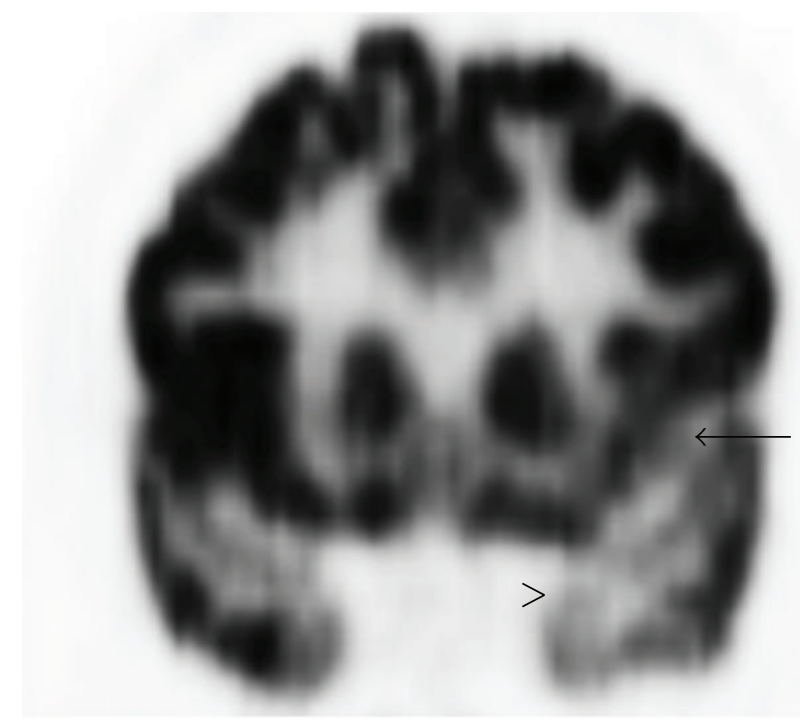

(e)

FIgURE 16: (Case 7) A 42-year-old woman with refractory CPS of suspected insular origin from the age of 32 years with left MTS on MRI. Her seizures would start with an aura as upper limb paraesthesia in about $20 \%$ of her seizures that would progress to lack of awareness and profound drooling lasting less than 30 seconds. Interictal EEG showing left temporal theta and left temporal-frontal spikes ((a) arrows and $(\mathrm{b}) *)((\mathrm{a})$ bipolar and (b) referential montage of same epoch). (c) EEG during her recorded seizures showing left temporal rhythmic theta after a brief attenuation for few seconds (c). FDG PET (d) and (e) demonstrate left insular (long arrow) and left mesial temporal (arrow head) hypometabolism ((c) and (d) arrows).

surgery. IEDs on surface EEG are more likely to correlate with the lateralization of the seizures in this situation. It is debatable if these subsets of patients need invasive study. In selected patients, noninvasive tests such as SPECT or PET may aid resective surgery without invasive monitoring.

\section{Postictal EEG}

Postictal EEG adds critical information particularly when seizure onset is unclear, or ictal changes are marred by muscle artifacts. The accuracy of postictal findings for lateralization has a higher degree of interrater reliability particularly in TLE than extratemporal seizures [90]. Postictal EEG findings include polymorphic lateralized delta activity [91], background suppression, and postictal spikes (57\%, 29\%, and 25\%, resp.) [92]. Postictal spikes are most sensitive for lateralization but may be affected by seizures spreading to the contralateral temporal lobe. In about a third, there may be no distinctive postictal change. These findings are affected by intensity of seizures, degree of HA, contralateral spread, and secondary generalization $[93,94]$. A combination of 


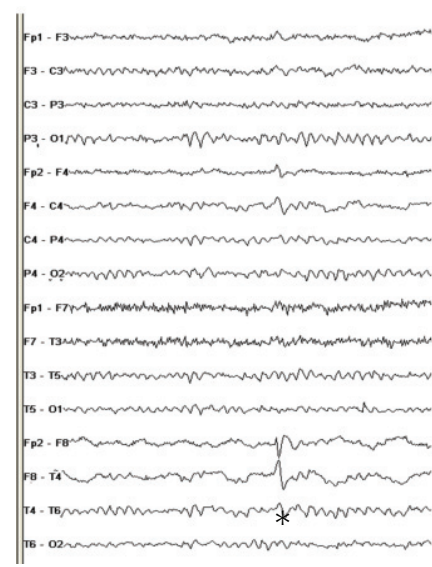

(a)

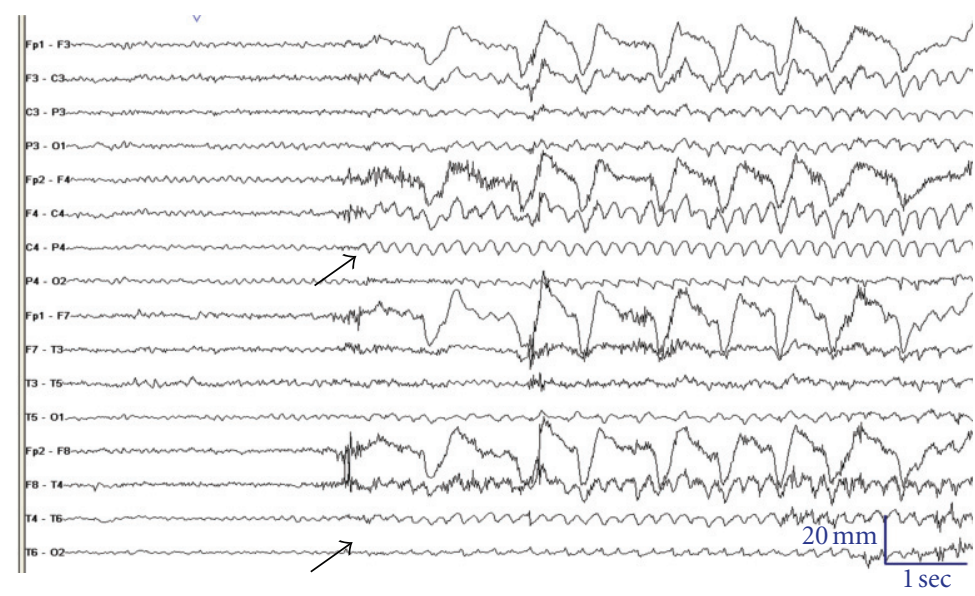

(b)

FIGURE 17: EEG in a young 25-year-old adult with right mesial occipital dysplasia who became seizure-free after lesionectomy. (a) Interictal EEG shows slowing over the right anterior temporal region and anterior temporal spikes $(*)$. (b) Ictal changes (arrow) were dominant over the right occipital region during one of his typical complex partial seizures. Comments: this case illustrates the presence of temporal spikes and slowing in a patient with occipital epilepsy due to a lesion (i.e., temporal lobe plus syndrome) [7-9].

postictal changes that persist longer is likely with widespread or secondary generalized seizure.

\section{High-Frequency Oscillations (HFOs)}

HFOs or ripples are electrical potentials in $80-600 \mathrm{~Hz}$ range recorded from the normal hippocampus and parahippocampal structures of humans with intracranial macroelectrodes. They may reflect normal inhibitory field potentials needed for neuronal synchronization. HFOs in the range of 250 $600 \mathrm{~Hz}$ (fast ripples, FRs) are often recorded from the pathologic hippocampus and parahippocampal structures of patients with mTLE [95]. They are prominent in the SOZ, and they provide independent additional information on epileptogenicity of IEDs [96]. HFOs have high specificity for SOZ even with very short recordings of only 10 minutes. Total resection of HFOs containing tissues results in good surgical outcomes $[97,98]$.

\section{Conclusions}

EEG remains the most important investigation in appropriately subclassifying patients with TLE along with other clinical and noninvasive data. Clinical history, physical findings, neuropsychological testing, MRI, and at times PET and SPECT, fMRI, or MEG data need to be integrated with EEG to select the ideal patients who would benefit from surgery. Most patients with TLE can be selected for surgery based on surface recordings alone. With discordant data, invasive monitoring helps to aid this decision.

\section{Acknowledgment}

The authors wish to acknowledge the Human Brain Tissue Repository (Brain Bank), Department of Neuropathology,
National Institute of Mental Health and Neurosciences, Bangalore for histological evaluation.

\section{References}

[1] S. Wiebe, W. T. Blume, J. P. Girvin, and M. Eliasziw, "A randomized, controlled trial of surgery for temporal-lobe epilepsy," New England Journal of Medicine, vol. 345, no. 5, pp. 311-318, 2001.

[2] R. A. Prayson, J. D. Reith, and I. M. Najm, "Mesial temporal sclerosis: a clinicopathologic study of 27 patients, including 5 with coexistent cortical dysplasia," Archives of Pathology and Laboratory Medicine, vol. 120, no. 6, pp. 532-536, 1996.

[3] L. M. Li, F. Cendes, F. Andermann et al., "Surgical outcome in patients with epilepsy and dual pathology," Brain, vol. 122, no. 5, pp. 799-805, 1999.

[4] F. Cendes, M. J. Cook, C. Watson et al., "Frequency and characteristics of dual pathology in patients with lesional epilepsy," Neurology, vol. 45, no. 11, pp. 2058-2064, 1995.

[5] J. Danckert, S. M. Mirsattari, F. Bihari, S. Danckert, A. A. Allman, and L. Janzen, "Functional MRI characteristics of a focal region of cortical malformation not associated with seizure onset," Epilepsy and Behavior, vol. 10, no. 4, pp. 615$625,2007$.

[6] W. T. Blume, J. Ravindran, and N. J. Lowry, "Late lateralizing and localizing EEG features of scalp-recorded temporal lobe seizures," Journal of Clinical Neurophysiology, vol. 15, no. 6, pp. 514-520, 1998.

[7] A. Patel, F. Alotaibi, W. T. Blume, and S. M. Mirsattari, "Independent component analysis of subdurally recorded occipital seizures," Clinical Neurophysiology, vol. 119, no. 11, pp. 2437-2446, 2008.

[8] W. T. Blume, S. E. Whiting, and J. P. Girvin, "Epilepsy surgery in the posterior cortex," Annals of Neurology, vol. 29, no. 6, pp. 638-645, 1991.

[9] W. T. Blume, S. Wiebe, and L. M. Tapsell, "Occipital epilepsy: lateral versus mesial,” Brain, vol. 128, no. 5, pp. 1209-1225, 2005. 
[10] P. Gloor, "Preoperative electroencephalographic investigation in temporal lobe epilepsy: extracranial and intracranial recordings," Canadian Journal of Neurological Sciences, vol. 18, no. 4, pp. 554-558, 1991.

[11] R. M. Sadler and J. Goodwin, "Multiple electrodes for detecting spikes in partial complex seizures," Canadian Journal of Neurological Sciences, vol. 16, no. 3, pp. 326-329, 1989.

[12] H. H. Jasper, "The ten-twenty electrode system of the International Federation," Electroencephalography and Clinical Neurophysiology, vol. 10, no. 10, pp. 371-375, 1958.

[13] W. J. Nowack, A. Janati, W. S. Metzer, and J. Nickols, "The anterior temporal electrode in the EEG of the adult," Clinical EEG Electroencephalography, vol. 19, no. 4, pp. 199-204, 1988.

[14] D. Silverman, R. Bernard, and M. Mellies, "The anterior temporal electrode and the ten-twenty system," Electroencephalography and Clinical Neurophysiology, vol. 12, no. 3, pp. 735-737, 1960.

[15] W. T. Blume, "The necessity for sphenoidal electrodes in the presurgical evaluation of temporal lobe epilepsy: con position," Journal of Clinical Neurophysiology, vol. 20, no. 5, pp. 305-310, 2003.

[16] G. E. Chatrian, E. Lettich, and P. L. Nelson, "Modified nomenclature for the "10\%" electrode system." Journal of Clinical Neurophysiology, vol. 5, no. 2, pp. 183-186, 1988.

[17] M. R. Sperling and J. Engel Jr, "Electroencephalographic recording from the temporal lobes: a comparison of ear, anterior temporal, and nasopharyngeal electrodes," Annals of Neurology, vol. 17, no. 5, pp. 510-513, 1985.

[18] M. R. Sperling, J. R. Mendius, and J. Engel Jr, "Mesial temporal spikes: a simultaneous comparison of sphenoidal, nasopharyngeal, and ear electrodes," Epilepsia, vol. 27, no. 1, pp. 81-86, 1986.

[19] M. Zijlmans, G. M. Huiskamp, A. C. van Huffelen, W. P. J. Spetgens, and F. S. S. Leijten, "Detection of temporal lobe spikes: comparing nasopharyngeal, cheek and anterior temporal electrodes to simultaneous subdural recordings," Clinical Neurophysiology, vol. 119, no. 8, pp. 1771-1777, 2008.

[20] G. Alarcón, N. Kissani, M. Dad et al., "Lateralizing and localizing values of ictal onset recorded on the scalp: evidence from simultaneous recordings with intracranial foramen ovale electrodes," Epilepsia, vol. 42, no. 11, pp. 1426-1437, 2001.

[21] D. Nilsson, M. Fohlen, C. Jalin, G. Dorfmuller, C. Bulteau, and O. Delalande, "Foramen ovale electrodes in the preoperative evaluation of temporal lobe epilepsy in children," Epilepsia, vol. 50, no. 9, pp. 2085-2096, 2009.

[22] T. R. Velasco, A. C. Sakamoto, V. Alexandre Jr et al., "Foramen ovale electrodes can identify a focal seizure onset when surface EEG fails in mesial temporal lobe epilepsy," Epilepsia, vol. 47, no. 8, pp. 1300-1307, 2006.

[23] A. Cherian, A. Radhakrishnan, S. Parameswaran, R. Varma, and K. Radhakrishnan, "Do sphenoidal electrodes aid in surgical decision making in drug resistant temporal lobe epilepsy," Clinical Neurophysiology, vol. 123, no. 3, pp. 463470, 2012.

[24] N. Kissani, G. Alarcon, M. Dad, C. D. Binnie, and C. E. Polkey, "Sensitivity of recordings at sphenoidal electrode site for detecting seizure onset: evidence from scalp, superficial and deep foramen ovale recordings," Clinical Neurophysiology, vol. 112, no. 2, pp. 232-240, 2001.

[25] A. M. Kanner, J. Parra, A. Gil-Nagel et al., "The localizing yield of sphenoidal and anterior temporal electrodes in ictal recordings: a comparison study," Epilepsia, vol. 43, no. 10, pp. 1189-1196, 2002.
[26] M. B. Hamaneh, C. Limotai, and H. O. Lüders, "Sphenoidal electrodes significantly change the results of source localization of interictal spikes for a large percentage of patients with temporal lobe epilepsy," Journal of Clinical Neurophysiology, vol. 28, no. 4, pp. 373-379, 2011.

[27] A. M. Kanner, L. Ramirez, and J. C. Jones, "The utility of placing sphenoidal electrodes under the foramen ovale with fluoroscopic guidance," Journal of Clinical Neurophysiology, vol. 12, no. 1, pp. 72-81, 1995.

[28] R. J. Wilkus and P. M. Thompson, "Sphenoidal electrode positions and basal EEG during long term monitoring," Epilepsia, vol. 26, no. 2, pp. 137-142, 1985.

[29] W. T. Blume, J. L. Borghesi, and J. F. Lemieux, "Interictal indices of temporal seizure origin," Annals of Neurology, vol. 34, no. 5, pp. 703-709, 1993.

[30] E. Pataraia, S. Lurger, W. Serles et al., "Ictal scalp EEG in unilateral mesial temporal lobe epilepsy," Epilepsia, vol. 39, no. 6, pp. 608-614, 1998.

[31] F. Cendes, L. M. Li, C. Watson, F. Andermann, F. Dubeau, and D. L. Arnold, "Is ictal recording mandatory in temporal lobe epilepsy? Not when the interictal electroencephalogram and hippocampal atrophy coincide," Archives of Neurology, vol. 57, no. 4, pp. 497-500, 2000.

[32] N. M. G. Bodde, J. L. Brooks, G. A. Baker et al., "Psychogenic non-epileptic seizures-definition, etiology, treatment and prognostic issues: a critical review," Seizure, vol. 18, no. 8, pp. 543-553, 2009.

[33] M. Pfänder, S. Arnold, A. Henkel et al., "Clinical features and EEG findings differentiating mesial from neocortical temporal lobe epilepsy," Epileptic Disorders, vol. 4, no. 3, pp. 189-195, 2002.

[34] H. M. Hamer, I. Najm, A. Mohamed, and E. Wyllie, "Interictal epileptiform discharges in temporal lobe epilepsy due to hippocampal sclerosis versus medial temporal lobe tumors," Epilepsia, vol. 40, no. 9, pp. 1261-1268, 1999.

[35] C. Aykut-Bingol, R. A. Bronen, J. H. Kim, D. D. Spencer, and S. S. Spencer, "Surgical outcome in occipital lobe epilepsy: implications for pathophysiology," Annals of Neurology, vol. 44, no. 1, pp. 60-69, 1998.

[36] N. Tandon, A. V. Alexopoulos, A. Warbel, I. M. Najm, and W. E. Bingaman, "Occipital epilepsy: spatial categorization and surgical management: clinical article," Journal of Neurosurgery, vol. 110, no. 2, pp. 306-318, 2009.

[37] F. Cendes, F. Dubeau, F. Andermann et al., "Significance of mesial temporal atrophy in relation to intracranial ictal and interictal stereo EEG abnormalities," Brain, vol. 119, no. 4, pp. 1317-1326, 1996.

[38] M. Sadler and R. Desbiens, "Scalp EEG in temporal lobe epilepsy surgery," Canadian Journal of Neurological Sciences, vol. 27, no. 1, pp. S22-S28, 2000.

[39] R. Schulz, H. O. Lüders, M. Hoppe, I. Tuxhorn, T. May, and A. Ebner, "Interictal EEG and ictal scalp EEG propagation are highly predictive of surgical outcome in mesial temporal lobe epilepsy," Epilepsia, vol. 41, no. 5, pp. 564-570, 2000.

[40] M. Y. Chung, T. S. Walczak, D. V. Lewis, D. V. Dawson, and R. Radtke, "Temporal lobectomy and independent bitemporal interictal activity: what degree of lateralization is sufficient?" Epilepsia, vol. 32, no. 2, pp. 195-201, 1991.

[41] A. Hufnagel, C. E. Elger, H. Pels et al., "Prognostic significance of ictal and interictal epileptiform activity in temporal lobe epilepsy," Epilepsia, vol. 35, no. 6, pp. 1146-1153, 1994.

[42] A. Koukou, S. Dupont, W. Szurhaj, M. Baulac, P. Derambure, and C. Adam, "Complete change of seizure and 
spike lateralization in temporal lobe epilepsy at two separate monitorings," Clinical Neurophysiology, vol. 118, no. 2, pp. 255-261, 2007.

[43] M. D. Holmes, A. N. Miles, C. B. Dodrill, G. A. Ojemann, and A. J. Wilensky, "Identifying potential surgical candidates in patients with evidence of bitemporal epilepsy," Epilepsia, vol. 44, no. 8, pp. 1075-1079, 2003.

[44] P. Halász, J. Janszky, G. Y. Rásonyi et al., "Postoperative interictal spikes during sleep contralateral to the operated side is associated with unfavourable surgical outcome in patients with preoperative bitemporal spikes." Seizure, vol. 13, no. 7, pp. 460-466, 2004.

[45] B. J. Steinhoff, N. K. So, S. Lim, and H. O. Luders, "Ictal scalp EEG in temporal lobe epilepsy with unitemporal versus bitemporal interictal epileptiform discharges," Neurology, vol. 45 , no. 5 , pp. $889-896,1995$.

[46] R. M. Sadler and W. T. Blume, "Significance of bisynchronous spike-waves in patients with temporal lobe spikes," Epilepsia, vol. 30, no. 2, pp. 143-146, 1989.

[47] P. Ryvlin and P. Kahane, "The hidden causes of surgeryresistant temporal lobe epilepsy: extratemporal or temporal plus?" Current Opinion in Neurology, vol. 18, no. 2, pp. 125$127,2005$.

[48] C. Barba, G. Barbati, L. Minotti, D. Hoffmann, and P. Kahane, "Ictal clinical and scalp-EEG findings differentiating temporal lobe epilepsies from temporal 'plus' epilepsies," Brain, vol. 130, no. 7, pp. 1957-1967, 2007.

[49] Y. Aghakhani, A. Rosati, F. Dubeau, A. Olivier, and F. Andermann, "Patients with temporoparietal ictal symptoms and inferomesial EEG do not benefit from anterior temporal resection," Epilepsia, vol. 45, no. 3, pp. 230-236, 2004.

[50] M. Guenot and J. Isnard, "Epilepsy and insula," Neurochirurgie, vol. 54, no. 3, pp. 374-381, 2008.

[51] P. Ryvlin, "Avoid falling into the depths of the insular trap," Epileptic Disorders, vol. 8, supplement 2, pp. S37-S56, 2006.

[52] J. Isnard, M. Guénot, M. Sindou, and F. Mauguière, "Clinical manifestations of insular lobe seizures: a stereo- electroencephalographic study," Epilepsia, vol. 45, no. 9, pp. 1079-1090, 2004.

[53] K. Ostrowsky, J. Isnard, P. Ryvlin, M. Guénot, C. Fischer, and F. Mauguière, "Functional mapping of the insular cortex: clinical implication in temporal lobe epilepsy," Epilepsia, vol. 41, no. 6, pp. 681-686, 2000.

[54] C. Munari, J. Talairach, A. Bonis et al., "Differential diagnosis between temporal and "perisylvian" epilepsy in a surgical perspective," Acta Neurochirurgica, vol. 30, pp. 97-101, 1980.

[55] J. S. Ebersole and P. B. Wade, "Spike voltage topography identifies two types of frontotemporal epileptic foci," Neurology, vol. 41, no. 9, pp. 1425-1433, 1991.

[56] C. Baumgartner, G. Lindinger, A. Ebner et al., "Propagation of interictal epileptic activity in temporal lobe epilepsy," Neurology, vol. 45, no. 1, pp. 118-122, 1995.

[57] R. Krendl, S. Lurger, and C. Baumgartner, "Absolute spike frequency predicts surgical outcome in TLE with unilateral hippocampal atrophy," Neurology, vol. 71, no. 6, pp. 413-418, 2008.

[58] M. Avoli, "Do interictal discharges promote or control seizures? Experimental evidence from an in vitro model of epileptiform discharge," Epilepsia, vol. 42, supplement 3, pp. 2-4, 2001.

[59] A. Rosati, Y. Aghakhani, A. Bernasconi et al., "Intractable temporal lobe epilepsy with rare spikes is less severe than with frequent spikes," Neurology, vol. 60, no. 8, pp. 1290-1295, 2003.
[60] O. Stüve, C. B. Dodrill, M. D. Holmes, and J. W. Miller, "The absence of interictal spikes with documented seizures suggests extratemporal epilepsy," Epilepsia, vol. 42, no. 6, pp. 778-781, 2001.

[61] F. Saito, Y. Fukushima, S. Kubota, and T. Sato, "Clinicoelectroencephalographical significance of small sharp spikes," Brain and Nerve, vol. 35, no. 3, pp. 221-227, 1983.

[62] J. D. Geyer, E. Bilir, R. E. Faught, R. Kuzniecky, and F. Gilliam, "Significance of interictal temporal lobe delta activity for localization of the primary epileptogenic region," Neurology, vol. 52, no. 1, pp. 202-205, 1999.

[63] J. Reiher, M. Beaudry, and C. P. Leduc, “Temporal intermittent rhythmic delta activity (TIRDA) in the diagnosis of complex partial epilepsy: sensitivity, specificity and predictive value," Canadian Journal of Neurological Sciences, vol. 16, no. 4, pp. 398-401, 1989.

[64] N. Dericioglu and S. Saygi, "Ictal scalp EEG findings in patients with mesial temporal lobe epilepsy," Clinical EEG \& Neuroscience, vol. 39, no. 1, pp. 20-27, 2008.

[65] S. Y. Lee, S. K. Lee, C. H. Yun, K. K. Kim, and C. K. Chung, "Clinico-electrical characteristics of lateral temporal lobe epilepsy, anterior and posterior lateral temporal lobe epilepsy," Journal of Clinical Neurology, vol. 2, no. 2, pp. 118125, 2006.

[66] J. S. Ebersole and S. V. Pacia, "Localization of temporal lobe foci by ictal EEG patterns," Epilepsia, vol. 37, no. 4, pp. 386399, 1996.

[67] N. Foldvary, N. Lee, G. Thwaites et al., "Clinical and electrographic manifestations of lesional neocortical temporal lobe epilepsy," Neurology, vol. 49, no. 3, pp. 757-768, 1997.

[68] W. T. Blume and M. Kaibara, "The start-stop-start phenomenon of subdurally recorded seizures," Electroencephalography and Clinical Neurophysiology, vol. 86, no. 2, pp. 94-99, 1993.

[69] C. Adam, "How do the temporal lobes communicate in medial temporal lobe seizures?" Revue Neurologique, vol. 162, no. 8-9, pp. 813-818, 2006.

[70] J. P. Lieb, R. M. Dasheiff, and J. Engel, "Role of the frontal lobes in the propagation of mesial temporal lobe seizures," Epilepsia, vol. 32, no. 6, pp. 822-837, 1991.

[71] C. E. Napolitano and M. Orriols, "Two types of remote propagation in mesial temporal epilepsy: analysis with scalp ictal EEG," Journal of Clinical Neurophysiology, vol. 25, no. 2, pp. 69-76, 2008.

[72] L. Eross, L. Entz, D. Fabó et al., "Interhemispheric propagation of seizures in mesial temporal lobe epilepsy," Ideggyógyászati Szemle, vol. 62, no. 9-10, pp. 319-325, 2009.

[73] P. D. Adelson, M. P. Black, J. R. Madsen et al., "Use of subdural grids and strip electrodes to identify a seizure focus in children," Pediatric Neurosurgery, vol. 22, no. 4, pp. 174$180,1995$.

[74] J. M. Johnston Jr, F. T. Mangano, J. G. Ojemann, S. P. Tae, E. Trevathan, and M. D. Smyth, "Complications of invasive subdural electrode monitoring at St. Louis Children's Hospital, 1994-2005," Journal of Neurosurgery, vol. 105, no. 5, pp. 343-347, 2006.

[75] A. M. Siegel, B. C. Jobst, V. M. Thadani et al., "Medically intractable, localization-related epilepsy with normal MRI: presurgical evaluation and surgical outcome in 43 patients," Epilepsia, vol. 42, no. 7, pp. 883-888, 2001.

[76] D. A. Steven, Y. M. Andrade-Souza, J. G. Burneo, R. S. McLachlan, and A. G. Parrent, "Insertion of subdural strip electrodes for the investigation of temporal lobe epilepsy. 
Technical note," Journal of Neurosurgery, vol. 106, no. 6, pp. 1102-1106, 2007.

[77] J. J. Van Gompel, F. B. Meyer, W. R. Marsh, K. H. Lee, and G. A. Worrell, "Stereotactic electroencephalography with temporal grid and mesial temporal depth electrode coverage: does technique of depth electrode placement affect outcome?" Journal of Neurosurgery, vol. 113, no. 1, pp. 32-38, 2010.

[78] D. G. Placantonakis, S. Shariff, F. Lafaille et al., "Bilateral intracranial electrodes for lateralizing intractable epilepsy: efficacy, risk, and outcome," Neurosurgery, vol. 66, no. 2, pp. 274-283, 2010.

[79] S. Eisenschenk, R. L. Gilmore, J. E. Cibula, and S. N. Roper, "Lateralization of temporal lobe foci: depth versus subdural electrodes," Clinical Neurophysiology, vol. 112, no. 5, pp. 836$844,2001$.

[80] W. T. Blume, G. M. Holloway, and S. Wiebe, "Temporal epileptogenesis: localizing value of scalp and subdural interictal and ictal EEG data," Epilepsia, vol. 42, no. 4, pp. 508-514, 2001.

[81] M. R. Sperling and M. J. O'Connor, "Comparison of depth and subdural electrodes in recording temporal lobe seizures," Neurology, vol. 39, no. 11, pp. 1497-1504, 1989.

[82] J. A. Ogren, A. Bragin, C. L. Wilson et al., "Three-dimensional hippocampal atrophy maps distinguish two common temporal lobe seizure-onset patterns," Epilepsia, vol. 50, no. 6, pp. 1361-1370, 2009.

[83] D. King and S. S. Spencer, "Invasive electroencephalography in mesial temporal lobe epilepsy," Journal of Clinical Neurophysiology, vol. 12, no. 1, pp. 32-45, 1995.

[84] A. L. Velasco, C. L. Wilson, T. L. Babb, and J. Engel Jr, "Functional and anatomic correlates of two frequently observed temporal lobe seizure-onset patterns," Neural Plasticity, vol. 7, no. 1-2, pp. 49-63, 2000.

[85] A. Bragin, C. L. Wilson, T. Fields, I. Fried, and J. Engel Jr, "Analysis of seizure onset on the basis of wideband EEG recordings," Epilepsia, vol. 46, supplement 5, pp. 59-63, 2005.

[86] S. A. Lee, D. D. Spencer, and S. S. Spencer, "Intracranial EEG seizure-onset patterns in neocortical epilepsy," Epilepsia, vol. 41, no. 3, pp. 297-307, 2000.

[87] P. Gloor, V. Salanova, A. Olivier, and L. F. Quesney, "The human dorsal hippocampal commissure. An anatomically identifiable and functional pathway," Brain, vol. 116, no. 5, pp. 1249-1273, 1993.

[88] F. Spanedda, F. Cendes, and J. Gotman, "Relations between EEG seizure morphology, interhemispheric spread, and mesial temporal atrophy in bitemporal epilepsy," Epilepsia, vol. 38, no. 12, pp. 1300-1314, 1997.

[89] S. Mintzer, F. Cendes, J. Soss et al., "Unilateral hippocampal sclerosis with contralateral temporal scalp ictal onset," Epilepsia, vol. 45, no. 7, pp. 792-802, 2004.

[90] T. S. Walczak, R. A. Radtke, and D. V. Lewis, "Accuracy and interobserver reliability of scalp ictal EEG," Neurology, vol. 42, no. 12, pp. 2279-2285, 1992.

[91] M. M. S. Jan, M. Sadler, and S. R. Rahey, "Lateralized postictal EEG delta predicts the side of seizure surgery in temporal lobe epilepsy," Epilepsia, vol. 42, no. 3, pp. 402-405, 2001.

[92] M. Kaibara and W. T. Blume, "The postictal electroencephalogram," Electroencephalography and Clinical Neurophysiology, vol. 70, no. 2, pp. 99-104, 1988.

[93] P. W. Olejniczak, E. Mader, G. Butterbaugh, B. J. Fisch, and M. Carey, "Postictal EEG suppression and hippocampal atrophy in temporal lobe epilepsy," Journal of Clinical Neurophysiology, vol. 18, no. 1, pp. 2-8, 2001.

[94] J. Janszky, A. Fogarasi, H. Jokeit, R. Schulz, M. Hoppe, and A. Ebner, "Spatiotemporal relationship between seizure activity and interictal spikes in temporal lobe epilepsy," Epilepsy Research, vol. 47, no. 3, pp. 179-188, 2001.

[95] J. Engel Jr, A. Bragin, R. Staba, and I. Mody, "High-frequency oscillations: what is normal and what is not?" Epilepsia, vol. 50, no. 4, pp. 598-604, 2009.

[96] J. Jacobs, P. LeVan, R. Chander, J. Hall, F. Dubeau, and J. Gotman, "Interictal high-frequency oscillations $(80-500 \mathrm{~Hz})$ are an indicator of seizure onset areas independent of spikes in the human epileptic brain," Epilepsia, vol. 49, no. 11, pp. 1893-1907, 2008.

[97] J. Y. Wu, R. Sankar, J. T. Lerner, J. H. Matsumoto, H. V. Vinters, and G. W. Mathern, "Removing interictal fast ripples on electrocorticography linked with seizure freedom in children," Neurology, vol. 75, no. 19, pp. 1686-1694, 2010.

[98] J. Jacobs, M. Zijlmans, R. Zelmann et al., "High-frequency electroencephalographic oscillations correlate with outcome of epilepsy surgery," Annals of Neurology, vol. 67, no. 2, pp. 209-220, 2010. 


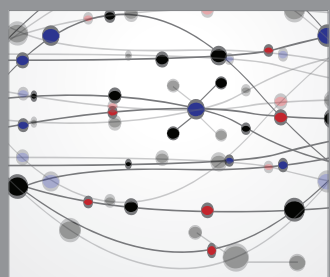

The Scientific World Journal
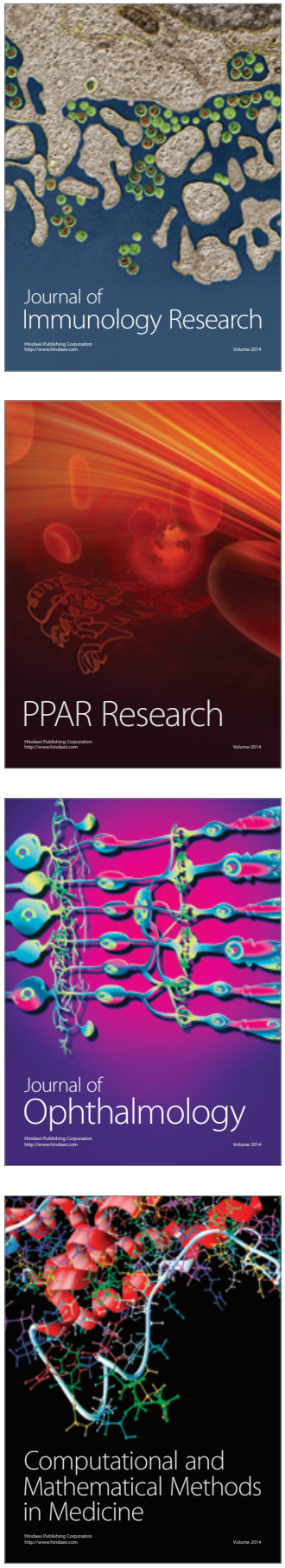

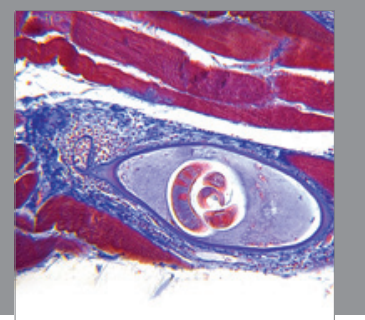

Gastroenterology

Research and Practice
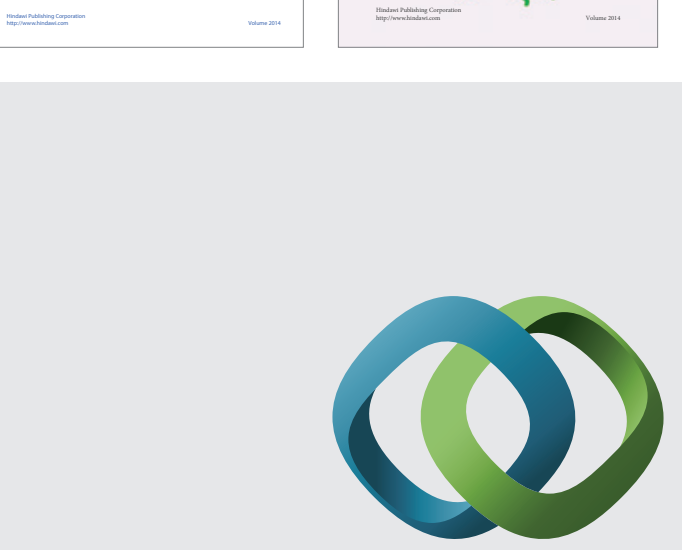

\section{Hindawi}

Submit your manuscripts at

http://www.hindawi.com
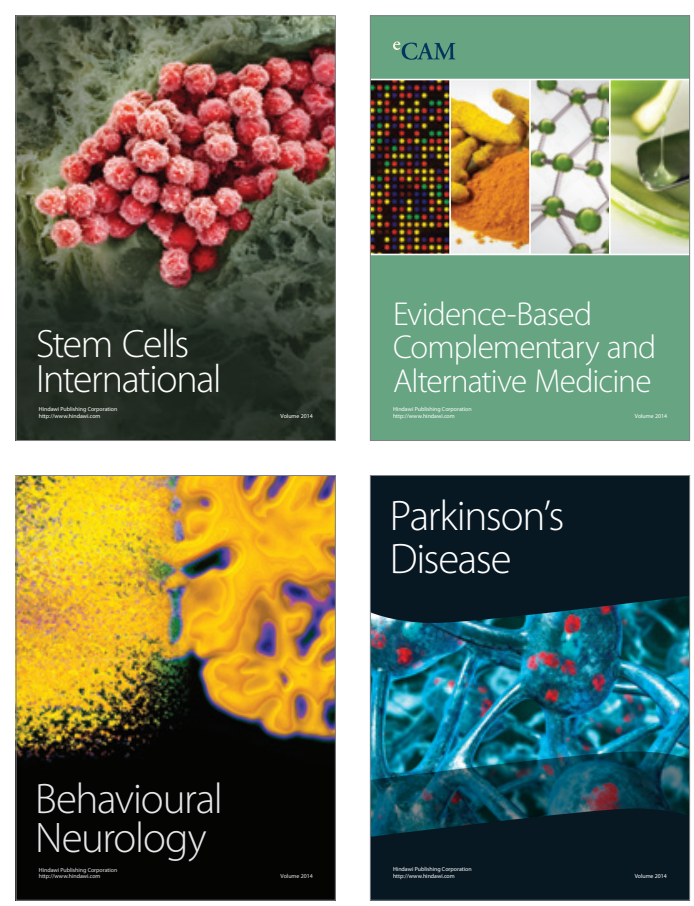

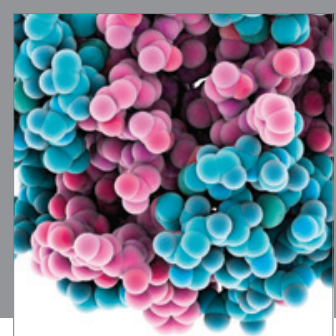

Journal of
Diabetes Research

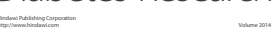

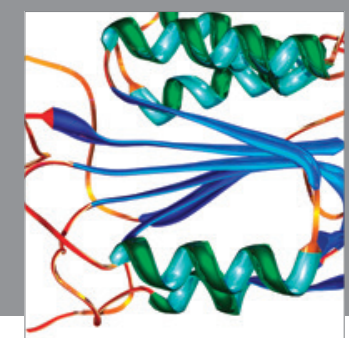

Disease Markers
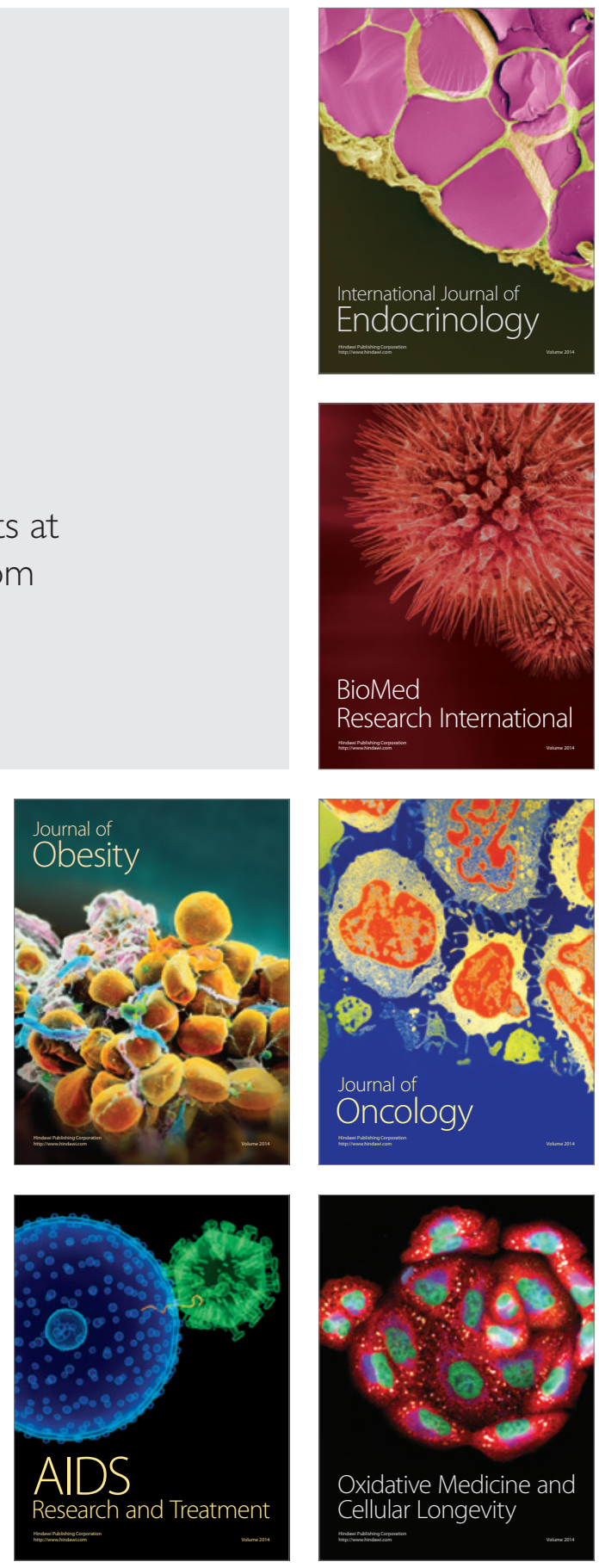